\title{
Evaluation of visual-induced motion sickness from head-mounted display using heartbeat evoked potential: a cognitive load-focused approach
}

\author{
Sangin Park ${ }^{1} \cdot$ Laehyun Kim $^{1,2} \cdot$ Jangho Kwon $^{1} \cdot$ Soo Ji Choi ${ }^{3} \cdot$ Mincheol Whang $^{4}$
}

Received: 8 April 2021 / Accepted: 20 October 2021 / Published online: 1 December 2021

(c) The Author(s) 2021

\begin{abstract}
Based on sensory conflict theory, motion sickness is strongly related to the information processing capacity or resources of the brain to cope with the multi-sensory stimuli experienced by watching virtual reality (VR) content. The purpose of this research was to develop a method of measuring motion sickness using the heart-evoked potential (HEP) phenomenon and propose new indicators for evaluating motion sickness. Twenty-eight undergraduate volunteers of both genders (14 females) participated in this study by watching VR content on both 2D and head-mounted devices (HMD) for $15 \mathrm{~min}$. The responses of HEP measures such as alpha power, latency, and amplitude of first and second HEP components were compared using paired t-tests and ANCOVA. This study confirmed that motion sickness leads to a decline in cognitive processing, as demonstrated by increasing in alpha power of HEP. Also, the proposed indicators such as latency and amplitude of the HEP waveform showed significant differences during the experience of motion sickness and exhibited high correlations with alpha power measures. Latencies of the first HEP component, in particular, are recommended as better quantitative evaluators of motion sickness than other measures, following the multitrait-multimethod matrix. The proposed model for motion sickness was implemented in a support vector machine with a radial basis function kernel, and validated on twenty new participants. The accuracy, F1 score, precision, recall, and area under the curve (AUC) of the motion-sickness classification results were 0.875 , $0.865,0.941,0.8$, and 0.962 , respectively.
\end{abstract}

Keywords Visual-induced motion sickness (VIMS) · Heartbeat evoked potential (HEP) · Head-mounted display (HMD) . Heart-brain synchronization · Cognitive load

\section{Introduction}

Mincheol Whang

whang@smu.ac.kr

Sangin Park

sipark@kist.re.kr

Laehyun Kim

laehyunk@kist.re.kr

Jangho Kwon

g15007@kist.re.kr

Soo Ji Choi

sooart0504@gmail.com

1 Center for Bionics, Korea Institute of Science and Technology, 5 Hwarang-ro 14-Gil, Seongbuk-gu, Seoul, Republic of Korea

2 Department of HY-KIST Bio-Convergence, Hanyang University, Seoul 04763, Republic of Korea
The recent emergence of virtual reality (VR) has contributed immensely to the advancement of technology and increased economic activity in this area. It has also shifted the focus of industry worldwide. In the past two decades, VR has been used in areas such as architecture, education and training, mobile technology, medical visualization, user interfaces, entertainment, and manufacturing (Azuma 1997; Höllerer et al. 1999; Zyda 2005; Pan et al. 2006; Van Krevelen and Poelman 2010; Kesim and Ozarslan 2012; Rodríguez et al.

3 Emphasis in Visual Communication Design, Bachelor of Fine Arts, School of the Art Institute of Chicago, $36 \mathrm{~S}$ Wabash, Chicago, IL 60603, USA

4 Department of Intelligent Engineering Information for Human, Sangmyung University, 7 Hongji-dong, Jongro-Ku, Seoul, Republic of Korea 
2015; Stolz et al. 2019). These integrations of technology have enhanced the efficiency and accuracy of tasks in the workplace and daily activities. VR technologies have been combined to create a hybrid of virtual and augmented experiences (Raajan et al. 2012). The technology is still undergoing rapid development and has attracted the most attention in all fields of work and study, particularly in creating virtual content for entertainment, therapies, training practices, applications, and so on (Zyda 2005; Kesim and Ozarslan 2012).

VR appears to have a positive effect on people because the technology improves the experience of immersion, realism, interactivity, and co-existence (Steuer 1992; Psotka 1995; Ryan 1999; Lambooij et al. 2007, 2009; Bailenson et al. 2008; Clemente et al. 2014). However, there are also side effects, especially from head-mounted displays (HMD) with stereoscopic views, which prevent daily or long-term use of the devices. The technology is known to cause visually induced motion sickness (VIMS) (Hettinger and Riccio 1992; Kennedy et al. 2010; Naqvi et al. 2013) and symptoms such as visual fatigue, anxiety, nausea, disorientation, abdominal discomfort, and oculomotor symptoms (MonWilliams et al. 1993; Lambooij et al. 2007, 2009; Diels et al. 2007; Bouchard et al. 2011; Carnegie and Rhee 2015). The severity of these adverse effects ranges from mild (some discomfort) to significant (distressing enough to prevent the user from carrying on with the experience). Some studies have even investigated the use of drugs such as hyoscine hydrobromide to relieve the associated motion sickness for a short period (Regan 1995; Regan and Ramsey 1996). This human factor may, ultimately, negatively influence the advancement and popularization of the VR industry. To eliminate this possibility, human factors such as VIMS need to be identified carefully and resolved (Bos et al. 2008). Numerous factors may trigger VIMS including postural instability, such as body swaying, and viewing conditions, such as gaze angle, fixation, retinal slip, and HMD fields of view (Smart et al. 2002; Yokota et al. 2005; Merhi et al. 2007; Diels et al. 2007; Bos et al. 2010; Moss and Muth 2011; Kim et al. 2018). The likelihood of these triggers to cause VIMS needs verification and guidelines for minimalizing VIMS are required to inform developers and users. Therefore, studies on standardized indicators that quantitatively measure VIMS must be conducted.

Previous studies have measured motion sickness using self-reporting, behavior, and physiological responses to improve the viewing experience of VR content. Selfreporting-based studies demonstrated that subjective rating scores from a simulator sickness questionnaire (SSQ) (Merhi et al. 2007; Sharples et al. 2008; Kiryu et al. 2008; Palmisano et al. 2017; Mazloumi Gavgani et al. 2018) and motion sickness susceptibility questionnaire (MSSQ) (Yokota et al. 2005; Kim et al. 2005; Nalivaiko et al. 2015; Chuang et al. 2016; Mazloumi Gavgani et al. 2018) increased when view- ers experienced motion sickness. Other researchers measured motion sickness using the Coriolis test (Zuzewicz et al. 2011; Malinska et al. 2015) and the Graybiel and Hamilton questionnaire (Ohyama et al. 2007). In measuring motion sickness through observations of the viewer's behavior, including head, body, and eye movements, Merhi et al. (2007) reported that movements measured from six positions in the $X, Y$, and $Z$ axes of the head increased significantly when the subjects experienced motion sickness. In the study by Yokota et al. (2005), body sway (in the $X$ and $Y$ axes) during the presentation of a motion sickness-inducing stimulus was measured and was found to increase. Interestingly, another group reported that the area of the center of gravity (COG) tends to change from being elliptical to circular in shape when the subject experiences visually induced motion sickness (Chardonnet et al. 2015) and Kim et al. (2005) revealed that the rate of blinking is significantly positively correlated with the MSSQ score. Lastly, other researchers measured physiological responses to motion sickness such as heart rate, autonomic balance, respiration, and electroencephalogram (EEG) spectrum.

In terms of cardiac response, many previous studies found that an increased heart rate (Kim et al. 2005; Zuzewicz et al. 2011; Nalivaiko et al. 2015; Malinska et al. 2015) and activated sympathetic nervous system (i.e., increased low frequency (LF) and decreased high frequency (HF) on the heart rate variability (HRV) spectrum) (Uijtdehaage et al. 1992; Gianaros et al. 2003; Yokota et al. 2005; Ohyama et al. 2007; Kiryu et al. 2008; Zuzewicz et al. 2011; Malinska et al. 2015) are associated with motion sickness. In view of the activated sympathetic nervous system in response to motion sickness, one report mentions that skin temperature decreases and the galvanic skin response rises (Kim et al. 2005). Additionally, researchers have shown that respiration increases (Kim et al. 2005; Kiryu et al. 2008) and the spectral power of respiratory and blood pressure is activated (Kiryu et al. 2008) when motion sickness is being experienced. In studies related to brain response, motion sickness was assessed by brain activity observed on an EEG spectrum. Chuang et al. (2016) reported that increased motion sickness is correlated with the activation of alpha and gamma bands in motor, parietal, and occipital areas. The demonstrations of these phenomena have revealed an increase in the neurophysiological demand to process information from multi-modal sensory systems. Lin et al. (2013) showed that brain areas in the left and right motor, parietal, lateral, and midline occipital lobes are activated more than other brain areas while subjects are experiencing motion sickness. Additionally, the relative delta power increases and the relative beta power decreases in F3 and T3 regions (Kim et al. 2005). A summary of the literature associated with measuring motion sickness is given in Table 1. 
Table 1 The summary of literature for the motion sickness measurement

\begin{tabular}{|c|c|c|c|c|c|}
\hline Measurement & & Platform & Content & Participants & Paper \\
\hline \multirow[t]{14}{*}{ Subjective rating } & \multirow[t]{5}{*}{ SSQ } & \multirow[t]{3}{*}{ HMD } & Optic flow video & 13 & Palmisano et al. (2017) \\
\hline & & & Virtual factory & 139 & Sharples et al. (2008) \\
\hline & & & Console video game & 32 & Merhi et al. (2007) \\
\hline & & \multirow[t]{2}{*}{ Projector } & Stereoscopic vision & 17 & Chardonnet et al. (2015) \\
\hline & & & $\begin{array}{l}\text { First-person viewpoint } \\
\text { video }\end{array}$ & 27 & Kiryu et al. (2008) \\
\hline & \multirow[t]{6}{*}{ MSSQ } & \multirow[t]{2}{*}{ HMD } & Rollercoaster & 26 & Nalivaiko et al. (2015) \\
\hline & & & Virtual living room & 15 & Yokota et al. (2005) \\
\hline & & \multirow[t]{3}{*}{ Projector (simulator) } & Driving & 19 & Chuang et al. (2016) \\
\hline & & & Driving & 17 & Lin et al. (2013) \\
\hline & & & Driving & 7 & Yu et al. (2010) \\
\hline & & Projector & $3 \mathrm{D}$ virtual environment & 61 & Kim et al. (2005) \\
\hline & \multirow[t]{2}{*}{ Coriolis test } & HMD (simulator) & Forklift & 24 & Zuzewicz et al. (2011) \\
\hline & & HMD & Virtual work station & 19 & Malinska et al. (2015) \\
\hline & $\begin{array}{l}\text { Questionnaire of } \\
\text { Graybiel and } \\
\text { Hamilton }\end{array}$ & Projector & Texture pattern video & 10 & Ohyama et al. (2007) \\
\hline \multirow[t]{4}{*}{ Behavior } & Head movement & HMD & Console video game & 32 & Merhi et al. (2007) \\
\hline & \multirow[t]{2}{*}{ Body movement } & HMD & Virtual living room & 15 & Yokota et al.,(2005) \\
\hline & & Projector & Stereoscopic vision & 17 & Chardonnet et al. (2015) \\
\hline & Eye blink & Projector & $3 \mathrm{D}$ virtual environment & 61 & Kim et al. (2005) \\
\hline \multirow[t]{18}{*}{ Physiological response } & \multirow[t]{4}{*}{ HR } & HMD (simulator) & Forklift & 24 & Zuzewicz et al. (2011) \\
\hline & & \multirow[t]{2}{*}{ HMD } & Virtual work station & 19 & Malinska et al. (2015) \\
\hline & & & Rollercoaster & 26 & Nalivaiko et al. (2015) \\
\hline & & Projector & $3 \mathrm{D}$ virtual environment & 61 & Kim et al. (2005) \\
\hline & \multirow{5}{*}{$\begin{array}{l}\text { Autonomic balance (LF, } \\
\text { HF) }\end{array}$} & HMD (simulator) & Forklift & 24 & Zuzewicz et al. (2011) \\
\hline & & HMD & Virtual work station & 19 & Malinska et al. (2015) \\
\hline & & & Virtual living room & 15 & Yokota et al. (2005) \\
\hline & & Projector & $\begin{array}{l}\text { First-person viewpoint } \\
\text { video }\end{array}$ & 27 & Kiryu et al. (2008) \\
\hline & & & Texture pattern video & 10 & Ohyama et al. (2007) \\
\hline & \multirow[t]{2}{*}{$\begin{array}{l}\text { Respiration (RR, HF, } \\
\text { RSA) }\end{array}$} & \multirow[t]{2}{*}{ Projector } & $\begin{array}{l}\text { First-person viewpoint } \\
\text { video }\end{array}$ & 27 & Kiryu et al. (2008) \\
\hline & & & $3 \mathrm{D}$ virtual environment & 61 & Kim et al. (2005) \\
\hline & Blood pressure (LF) & Projector & $\begin{array}{l}\text { First-person viewpoint } \\
\text { video }\end{array}$ & 27 & Kiryu et al. (2008) \\
\hline & \multirow[t]{2}{*}{ Temperature } & HMD & Rollercoaster & 26 & Nalivaiko et al. (2015) \\
\hline & & Projector & $3 \mathrm{D}$ virtual environment & 61 & Kim et al. (2005) \\
\hline & $\begin{array}{l}\text { Skin conductance, } \\
\text { gastric } \\
\text { tachyarrhythmia }\end{array}$ & Projector & $3 \mathrm{D}$ virtual environment & 61 & Kim et al. (2005) \\
\hline & $\begin{array}{l}\text { Motor, parietal, } \\
\text { occipital alpha, } \\
\text { gamma }\end{array}$ & \multirow[t]{2}{*}{ Projector (simulator) } & Driving & 19 & Chuang et al. (2016) \\
\hline & $\begin{array}{l}\text { Left and right motor, } \\
\text { parietal, lateral and } \\
\text { midline occipital }\end{array}$ & & Driving & 17 & Lin et al. (2013) \\
\hline & $\begin{array}{l}\text { F3, T3-delta } \\
\text { F3-slow Beta } \\
\text { T3-beta }\end{array}$ & Projector & $3 \mathrm{D}$ virtual environment & 61 & Kim et al. (2005) \\
\hline
\end{tabular}


The evaluation of motion sickness has thus far been scored subjectively according to predefined questionnaires and described using interview techniques. However, these questionnaires depend on personal interpretation and experience (Cain 2007). Since there are individual differences in interpretation, the repeatability and validity of subjective evaluations are often unclear and uncertain (Annett 2002; Cain 2007). Therefore, subjective rating is limited in that it cannot be used to assess motion sickness quantitatively. Moreover, the measurement of behavior using cameras or other devices is strongly influenced by the noise of the surrounding lights and movement. Also, the results of these measurements do not consider physiological mechanisms sufficiently to enable the phenomenon of motion sickness to be interpreted accurately. Motion sickness is strongly related to the subject's capacity to process information and resources for interpreting multi-sensory stimuli that are produced by VR content (Lin et al. 2007, 2013; Chen et al. 2010; Chuang et al. 2016). The cause of VIMS needs to be interpreted by cognitive load, i.e., the large amount of information from VR content that the brain needs to process compared with that from 2D display content. Thus, psychophysiological measures are the objective method of assessing motion sickness through consideration of psychological processes rather than behavioral and subjective rating measures. However, the cognitive process is not controlled by brain function alone but does involve the phenomenon of heartbeat evoked potential (HEP). The vagus nerve in the heart and major organs communicates sensory information from the external environment to the brain through efferent and afferent pathways (Davis and Natelson 1993; Porges 1997, 2007). The heart's response to sensory input has an effect on brain sensory systems such as emotional state, cognitive function, and performance (Hansen et al. 2003; McCraty et al. 2009; Park et al. 2014, 2015). Thus, there is a limitation to interpreting the phenomenon of VIMS from fragmentary responses in the heart or brain.

The purpose of this study was to determine a method for measuring motion sickness from VR content in HMD based on the HEP phenomenon and to propose new indicators for the evaluation of motion sickness. To assess motion sickness, HEP measurements (defined in Figs. 1, 5) taken from ECG and EEG signals collected while subjects viewed HMD content were compared with the same measurements made while subjects viewed $2 \mathrm{D}$ content. The proposed new indicators (latency and amplitude in HEP) in this study were compared with one (alpha power of HEP) used in a previous study (Park et al. 2015) by employing the multitraitmultimethod (MTMM) matrix for evaluating the reliability of variables Finally, selecting the useful classification features giving significant results in the HEP measurements, the motion-sickness and normal states were distinguished by various classifiers: linear support-vector machine (SVM), radial basis function (RBF) SVM, elastic net regularization, logistic regression (the LASSO model), and L2 (Ridge model) regularization. The accuracies, F1 scores, precisions, recalls, and areas under the curve (AUCs) were compared among the results of the various classifiers.

\section{Heartbeat evoked potential}

Heartbeat Evoked Potential (HEP) is a change in alpha brain waves to communicate the change of cardiac output such as blood pressure, heart rhythm, and variability from major organs such as heart to brain (Schandry and Montoya 1996). The communication occurs through a visceral nerve, known as "vagus nervous", to transmit visceral-afferent information into various brain parts: hypothalamic and thalamic nuclei, amygdalae, hippocampus, cerebellum, somatosensory cortex, prefrontal cortex, and insula (Warner and Cox 1962; Montoya et al. 1993; Davis and Natelson 1993; Drew et al. 2008). First, the visceral nerve transmits the information to the NTS (nucleus tractus solitarius) in the brainstem. The joint information at the NTS is then sent to the mid-brain such as hypothalamus and thalamus, followed by the arrival at the cortex (Janig 1996). The mid-brain transfers information with neocortex, especially at the frontal and prefrontal brain cortices. Some researchers have focused their studies on the premotor and orbital areas of the frontal lobe because of the direct processing of information through visceralafferent pathways from the mid-brain (hypothalamus and thalamus) to the prefrontal and frontal cortices (Nauta et al. 1986; Fuster 1988; Nieuwenhuys et al. 2007). These areas are related with attention and mental processes (Boussaoud 2001; Hartikainen and Knight 2003; Villena-Gonzalez et al. 2017), thus signifying the relationship between heartbeat and attention. Based on this relationship, a significant post-Rwave (250-450 ms) in Fz brain region was found (Schandry et al. 1986). Also, a negative shift was found in the range of 250-400 ms waveform at Fz, F7, F8, and Cz brain regions (Schandry and Weitkunat 1990). This significant change in HEP can be an indicator for assessing cortical activity such as attention and mental state by measuring the brain and heart synchronization (Pollatos and Schandry 2004; Fukushima et al. 2011; Lechinger et al. 2015).

A synchronization of heart and brain causes the brain to display a corresponding negative peak on the electroencephalography (EEG) simultaneously with the R-peak of the ECG signal. This synchronized response has previously been characterized as the event-related potential (ERP), and known to be related to the first and second periods of the HEP (McCraty et al. 2009; Park et al. 2015). The first period of HEP, 50-250 ms after the R-peak, reflects the time interval of the rate of change from heart to brain along the afferent pathway. When brain has more information to process, the 


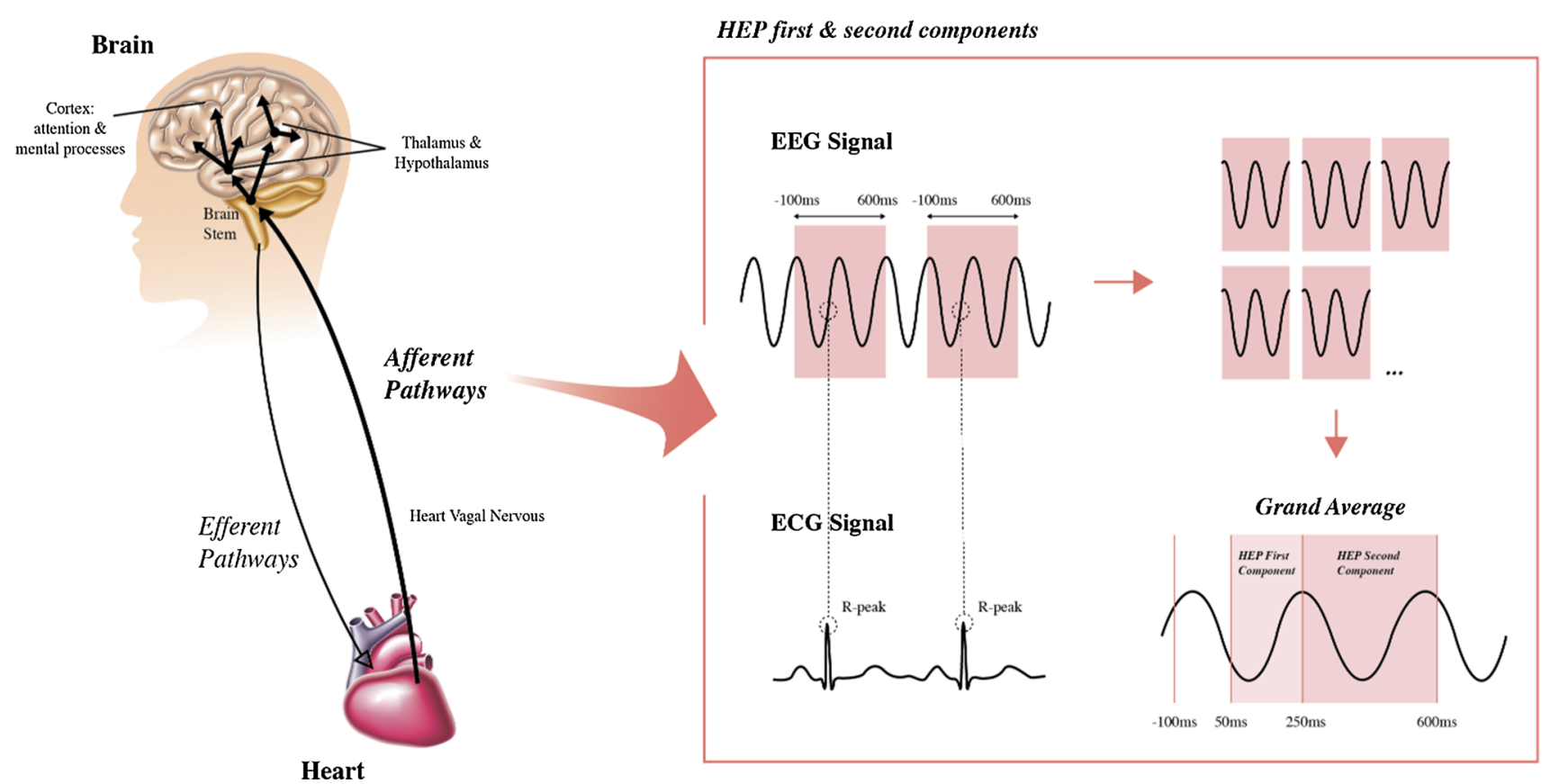

Fig. 1 Overview for heartbeat evoked potential (HEP)

heart-brain communication increases by the afferent pathway, and the synchronization in the alpha band also increases (Wölk et al. 1989; McCraty et al. 2009; Park et al. 2015). The second period of HEP, 250-600 ms after R-peak, indicates communication of both afferent signals and the hydraulic blood pressure wave from heart to brain, as well as alpha synchronization. An increased synchronization of the alpha wave in first period of HEP indicates an active state of cardiovascular information processing in the afferent pathway (Wölk et al. 1989; McCraty et al. 2009; Park et al. 2015). As shown in Fig. 1, HEP is a characteristic phenomenon from the synchronization of heart and brain through afferent pathways in the vagus nerve.

\section{Methods}

\subsection{Participants}

A total of 48 undergraduate volunteers of both genders ( 24 females) with ages ranging between 21 and 30 years (mean age, $24.95 \pm 2.69$ years) participated in this study. First, 28 participants (mean age, $25.04 \pm 2.22$ years) of both genders (14 females) were recruited to conduct statistical analysis. We have trained classifiers for using statistically significant features. Finally, 20 participants (mean age, $24.86 \pm 3.16$ years) of both genders (10 females) were recruited as test samples to validate the classifiers. All participants were right-handed and had no family or medical history of cardiovascular, autonomic, or central nervous system disorders. Every participant was asked to abstain from alcohol, cigarettes, and caffeine for $24 \mathrm{~h}$ prior to the experiment and to sleep normally. Consents from all participants, who were notified of the restrictions and requirements, were received. This research complied with the tenets of the Declaration of Helsinki and was approved by the Institutional Review Board at Sangmyung University (No. BE2018-46). Informed consent was obtained from each participant.

\subsection{Experimental procedure}

A pre-task was conducted to measure the sensitivity of participants to motion sickness prior to the main task. The participants were required to view the VR content "Ultimate Booster Experience" (GexagonVR, 2016) through an HTC VIVE device (HTC Inc., Taiwan \& Valve Inc., USA) for $10 \mathrm{~min}$ and report their motion sickness using a subjective rating and interview. The subjective rating was composed of three factors including nausea (7 items), oculomotor symptoms (7 items), and disorientation (7 items) and is well-known as the SSQ (Kennedy et al. 1993). The participants were also asked to self-report subjective motion sickness using a four-point scale (0-3) for 16 items both before and after viewing the VR content with the following specifications: (1) nausea (general discomfort, increased salivation, sweating, nausea, difficulty concentrating, stomach awareness, and burping); (2) oculomotor symptoms (general discomfort, fatigue, headache, eyestrain, difficulty focusing, difficulty concentrating, and blurred vision); and (3) disorientation [difficulty focusing, nausea, fullness of head, blurred 
vision, dizzy (eyes open), dizzy (eyes close), and vertigo]. The subjective rating of motion sickness was calculated by the SSQ score of the following Eq. (1) (Kennedy et al. 1993):

$$
\begin{aligned}
\text { SSQ score }= & \{(N \times 9.54)+(O \times 7.58)+(D \times 13.92)\} \\
& \times 3.74
\end{aligned}
$$

where the values of $N, O$, and $D$ were calculated by the summation of each of the items for nausea, oculomotor symptoms, and disorientation, respectively. Participants who did not feel or felt motion sickness severely enough to discontinue the experiment were excluded from the main experiment. Forty subjects participated in the pre-task; twenty-eight subjects were included in the main experiment.

They watched the 2D version of the VR content on the first day and the HMD version on the following day or vice versa. Because this study was designed "within subject design", the order of tasks (i.e., 2D and HMD) was randomly decided based on a counterbalanced repeated measures design to minimize sequence/order effects. The content of "No Limits 2 Roller Coaster Simulation" (Ole Lange, Mad Data GmbH \& Co. KG, 2014) was used in the experiment to cause motion sickness in participants; there were differences in 2D and VR versions only; however, the scene was identical. Participants watched both 2D and HMD versions using an LED monitor and HMD device, respectively. The VR contents used for the pre- and main tasks are shown in Fig. 2.

The 2D and HMD versions were presented on a 27-inch LED monitor (27MP68HM, LG) and HTC VIVE device (HTC Inc., Taiwan \& Valve Inc., USA). The participants were required to self-report subjective motion sickness using a 1 to 7 points scale in the SSQ both before and after the main experiment. A reference section was included for $5 \mathrm{~min}$ before and after the VR content. ECG and EEG signals were measured both before and after each VR content viewing period. The setup of the experiment procedure and environment are shown in Figs. 3 and 4.

\subsection{Data acquisition and signal processing}

EEG signals were recorded at a $500 \mathrm{~Hz}$ sampling rate from three channels on the scalp at positions FP1, FPz, and FP2 based on the international "10-10" system (ground: FAz, reference: average between electrodes on the two ears, amplitude: $70 \mu \mathrm{V}$, and DC level: $0-150 \mathrm{~Hz}$ ) and using a Mitsar-EEG 202 machine (Mitsar Inc., Russia). The electrode impedance was kept below $3 \mathrm{k} \Omega$. The FP1, FPz, and FP2 regions were measured because these regions were strongly related to the HEP phenomenon (Montoya et al. 1993; McCraty et al. 2009). ECG signals were recorded at a $500 \mathrm{~Hz}$ sampling rate using an amplifier system (ECG 100C amplifiers in BIOPAC system Inc., USA) based on the Lead-I method. The ECG signal was digitized with the DAQ-Board
(NI-DAQ-Pad9205 in National Instrument Inc., USA) and MP100 power supply (BIOPAC Systems Inc., USA).

The processing of HEP signals was as follows: (1) the ocular and muscular artifacts were removed from the EEG signals by artifact subspace reconstruction (Mullen et al. 2013); (2) the R-peak was detected from the ECG signals based on the QRS detection algorithm (Pan and Tompkins 1985); (3) EEG signals with the artifacts removed were separated from 50 to $600 \mathrm{~ms}$ based on R-peak; (4) these separated signals were averaged by the "grand average technique," and this signal was defined as the HEP signal in this study; (5) HEP signals were then divided into the two components of interest-the first period in HEP $(50-250 \mathrm{~ms}$ after the R-peak) and the second period in HEP (250-600 ms after the R-peak). The indicators of amplitude, latency, and alpha power in HEP were as follows. The amplitude of HEP was defined by the difference in value between positive and negative dominant peaks from the HEP signal in the range $50-600 \mathrm{~ms}$. The latency of the first and second components of HEP was defined by the location (time value) of the dominant positive peak from each period. The alpha power of the HEP first and second components was defined by the relative power of the alpha band from each period based on fast Fourier transform (FFT) (Wölk et al. 1989; McCraty et al. 2009; Park et al. 2019). Signal processing and the definitions of indicators are shown in Fig. 5.

\subsection{Statistical analysis}

This study was designed to test and compare the viewer's experience of motion sickness while experiencing both 2D and HMD contents "within subject design." Therefore, a paired $t$-test was performed on sample data based on the normality test. In addition, because the independent $t$-test could not confirm the viewer's state before watching the VR content, this study was also applied to an analysis of covariance (ANCOVA). The ANCOVA compared dependent variables between groups after the VR content with the pre-VR content baseline as a covariate (Keselman et al. 2016; McGibbon and Krebs 2004; Park et al. 2014; Mun et al. 2014). The statistical significance was controlled by the Bonferroni correction to resolve the problem caused by multiple comparison based on the number of each individual hypothesis (i.e., $\alpha$ $=0.05 / n)$ (Dunnett 1955). For this experiment, the statistically significant level of the HEP measure was set to 0.0033 (HEP indicators: alpha power (6), amplitude (3), and latency (6), $\alpha=0.05 / 15$ ). The effect size based on Cohen's $d$ (Morris et al. 2014) and the partial eta-squared value $\left(\eta_{p}^{2}\right)$ (de Morree et al. 2014) were calculated to confirm not only the statistical significance, but also the effect size. In the case of Cohen's $d$, standard values of $0.10,0.25$, and 0.40 for effect size were generally regarded as small, medium, and large, respectively. In the case of the partial eta-squared value, 
Fig. 2 The VR contents used for the pre- and main tasks. Top image: VR content used in the pre-task ("Ultimate Booster Experience," GexagonVR, 2016). Bottom image: VR content used in the main task ("No Limits 2 Roller Coaster Simulation," Ole Lange, Mad Data GmbH \& Co. KG, 2014)
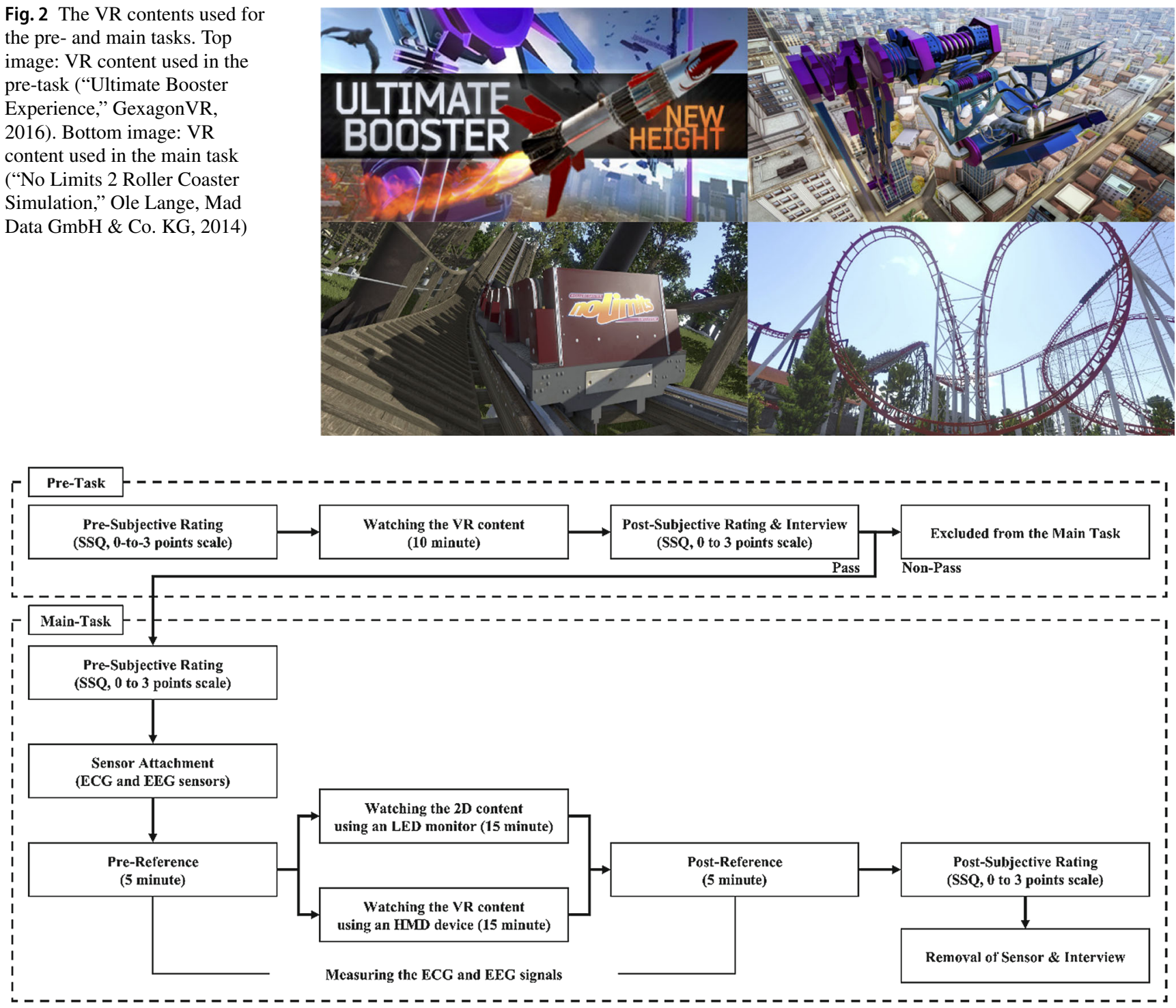

Fig. 3 The experimental procedure

standard values of $0.01,0.06$, and 0.14 for effect size were generally regarded as small, medium, and large, respectively (Huck et al. 1974). Also, the MTMM matrix was applied to verify test-retest reliability, convergent, and discriminant validity among various motion sickness indicators such as SSQ score, amplitude, alpha power, and latency of HEP in the FP1, FPz, and FP2 regions. If data samples involve the multi-trait and the multi-method, the MTMM matrix evaluates the relationship between multiple measures. This study defined the multi-trait and the multi-method as HEP measures and display types (2D and HMD), respectively. By confirming the monomethod-monotrait (reliability diagonal), monomethod-heterotrait, and heteromethod-monotrait, the test-retest reliability, discriminant validity, and convergent validity were tested and verified (Campbell and Fiske 1959). All statistical data analysis (i.e., subjective ratings,
HEP measures, and MTMM matrix) was conducted using IBM SPSS Statistics 21.0 for Windows (SPSS Inc., USA).

\subsection{Classification}

The classification into motion sickness and normal state was performed by five classification algorithms: SVM, RBF-SVM, elastic net regularization, LASSO model, and L2 (Ridge model) regularization. These algorithms were chosen for their popularity in biomedical data classification (Zhou et al. 2010; Herrera et al. 2013; Li et al. 2016). Fifteen HEP features (six alpha power features and six latency features of the HEP first and second components in the FP1, FP2, and FPz regions; three amplitudes of HEP waveforms in the FP1, FP2, and $\mathrm{FPz}$ regions) were extracted from our experimental data, and the ten statistical features 


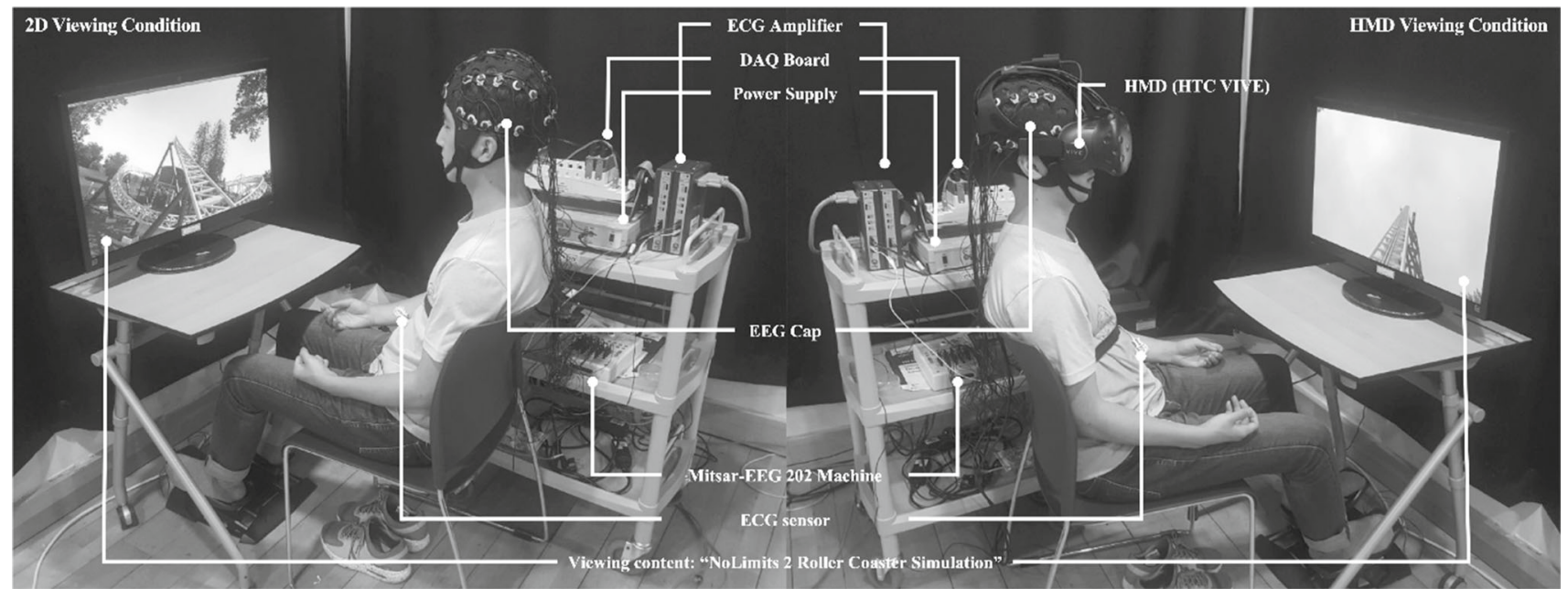

Fig. 4 The experimental environment and equipment

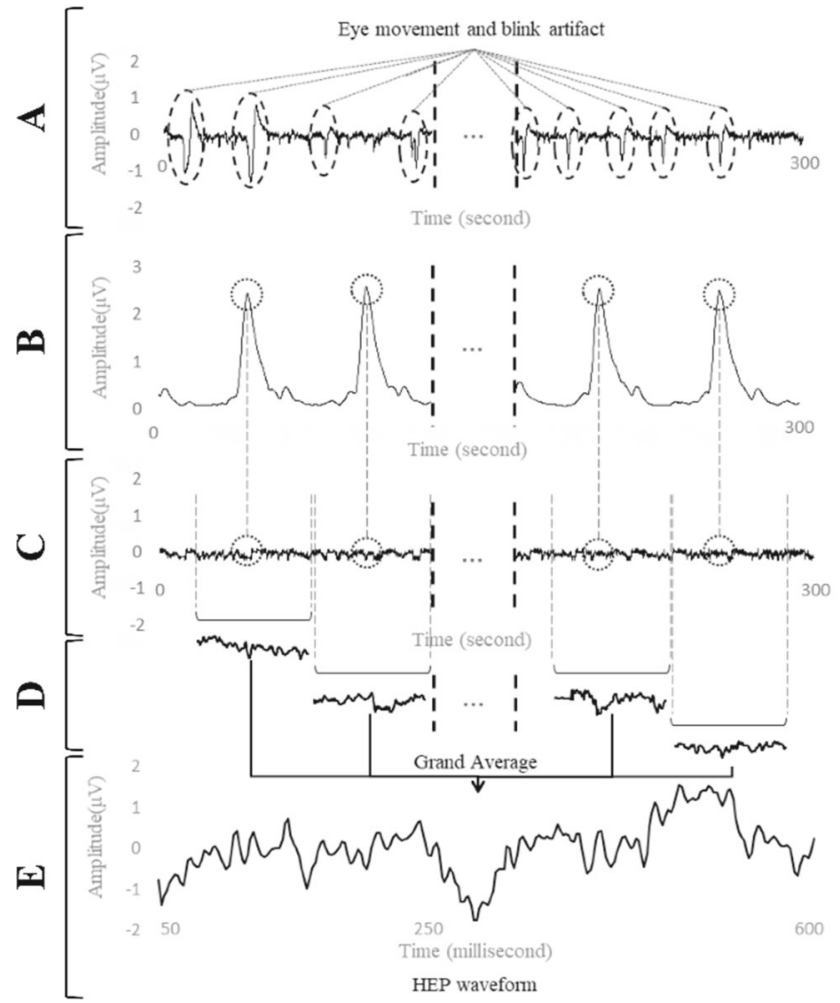

Fig. 5 Examples of signal processing for HEP measurements (alpha power, latency, and amplitude of HEP waveform). A Removing the eye movement and blinking artifacts from EEG signals. B Detecting the R-peak from ECG signals. C EEG signals after removing the artifacts.

showing statistically significant results were trained by the five-classification algorithm on a 28 -subject dataset. The classification performances of the five algorithms were evaluated by their accuracies, F1 scores, precisions, recalls, and AUCs (James et al. 2013; Saito and Rehmsmeier 2017) on a

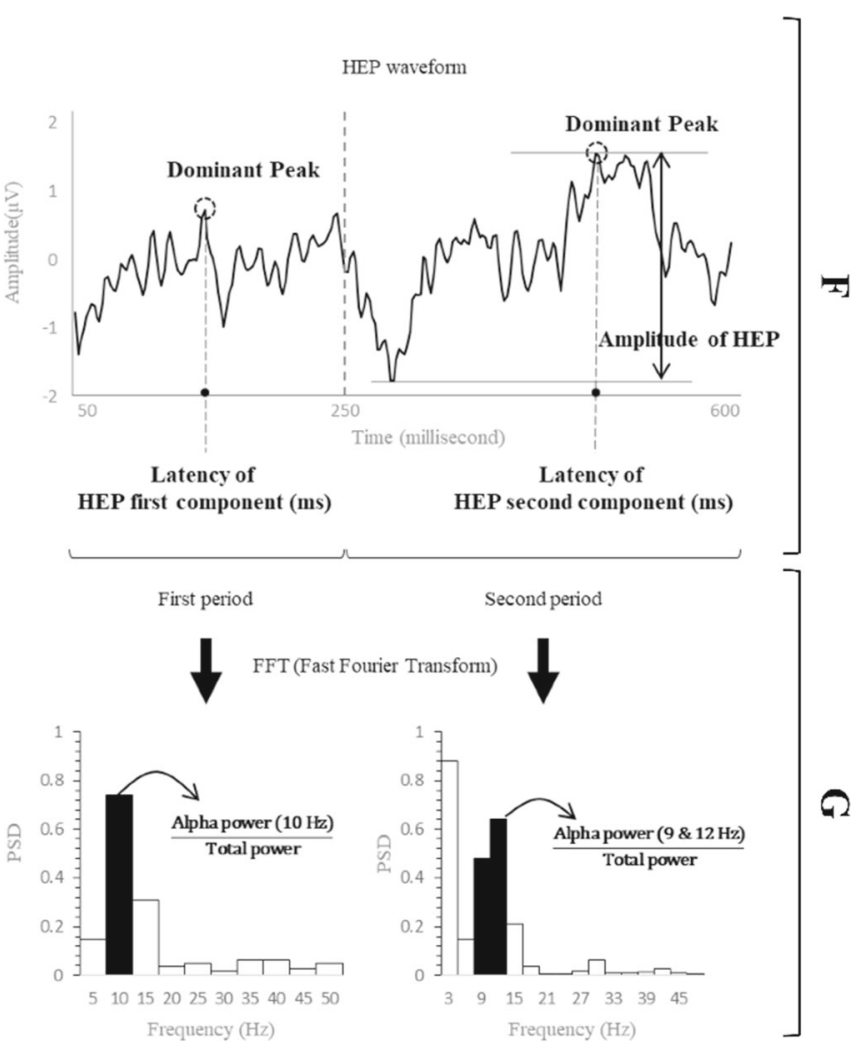

D Data separation (trial) in EEG signals based on R-peak from ECG signals. E Grand average signal for all trials in EEG signals. F Definition for latency of HEP first and second components and amplitude of HEP. G Definition for alpha power of HEP first and second components

new dataset of 20 subjects. The classification measures are defined below: 
Accuracy: proportion of correct predictions among the total number of predictions.

$$
\text { Accuracy }=(\mathrm{TP}+\mathrm{TN}) /(\mathrm{TP}+\mathrm{FN}+\mathrm{TN}+\mathrm{FP})
$$

Recall: ratio of correctly predicted positive observations to all observations in the actual class.

Recall $=\mathrm{TP} /(\mathrm{TP}+\mathrm{FN})$

Precision: ratio of correctly predicted positive observations to all predicted positive observations.

Precision $=\mathrm{TP} /(\mathrm{TP}+\mathrm{FP})$

F1 Score: weighted average of Precision and Recall. Note that this score accounts for both false positives and false negatives.

$\mathrm{F} 1$ Score $=2 *($ Recall $*$ Precision $) /($ Recall + Precision $)$

$A U C$ : area under the receiver operating characteristics (ROC) curve. The AUC value lies between 0.5 (bad classifier) and 1 (excellent classifier).

In the above expressions, TP and FN denote the numbers of correctly classified and incorrectly classified motionsickness instances, respectively, $\mathrm{TN}$ is the number of true negative classifications, and FP is the number of true positive classifications.

\subsubsection{Logistic regression: Lasso, Ridge, and ElasticNet}

Logistic regression is the appropriate analysis technique for dichotomous (binary) dependent variables. This linear classifier sums the weighted polynomials of features (Pereira et al. 2016). Logistic regression methods can be trained with different objective functions. For this purpose, we selected three objective functions: Lasso (Zhang et al. 2012), Ridge (Cessie and Houwelingen 1992), and ElasticNet (Zou and Hastie 2005), respectively.

\subsubsection{Support vector machine: linear and RBF}

SVM is widely used in physiological and biomedical data classification (Diykh and Li 2016). The SVM classifier finds the optimal hyperplane that maximizes the margin between two groups. The hyperplane is determined by the following decision function (Lima et al. 2009). This classifier determines the prediction class (positive if $f(x)$ exceeds 1 , negative if $f(x)$ is less than -1 ). We applied a linear SVM with no kernel (Chang et al. 2010), and SVM using an RBF kernel (Zhao et al. 2011).

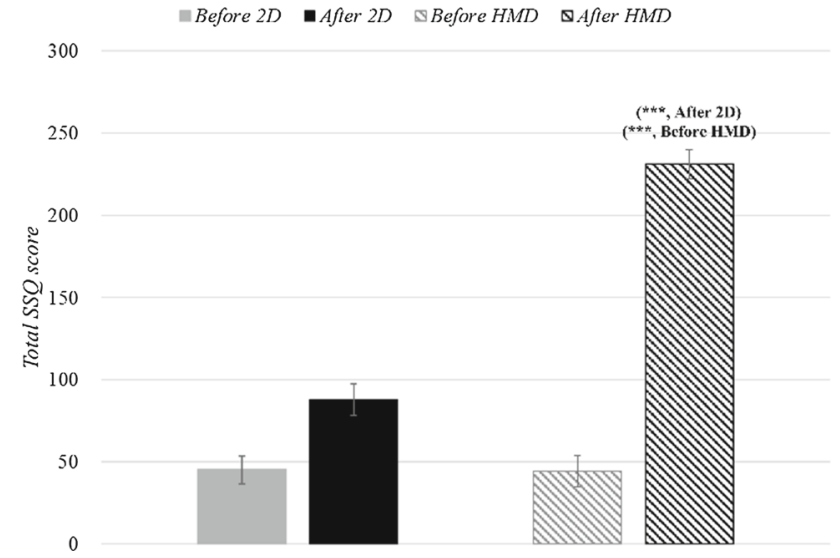

Fig. 6 Average subjective rating for motion sickness in the 2D and HMD conditions. There was a significant difference in the total SSQ score between the 2D and HMD groups based on a paired t-test and ANCOVA $(* p<0.05, * * p<0.01, * * * p<0.001)$

\section{Results}

\subsection{Subjective rating}

For the HMD viewing condition, a paired-samples $t$-test showed significant differences between the pre- and postviewing conditions for the total SSQ score $(t[54]=-10.801$, $p=0.0000$, with large effect size [Cohen's $d=2.940$ ]). However, no significant differences were found between the ratings obtained pre- and post-viewing in the $2 \mathrm{D}$ viewing condition $(t[54]=0.050, p=0.9604$, Cohen's $d=0.014)$, as shown in Fig. 6. ANCOVA was also performed to further compare the differences in the total SSQ scores between the 2D and HMD viewing conditions. There was a significant difference in the total SSQ score post-viewing with an adjusted total SSQ score in the pre-viewing condition as a covariate $(F[1,54]=127.989, p=0.0000$, with large effect size $\left.\left[\eta_{p}^{2}=0.707\right]\right)$, as shown in Fig. 6 .

\subsection{Alpha power, latency, and amplitude of HEP}

In assessing the results from the HMD viewing task, a pairedsamples $t$-test showed that the alpha power of the first HEP component post-viewing was significantly higher than that found before the viewing task in FP1 $(t[54]=-4.197, p$ $=0.0001$, with large effect size [Cohen's $d=1.142]$ ), FPz $(t[54]=-4.296, p=0.0000$, with large effect size [Cohen's $d=1.169])$, and FP2 $(t[54]=-4.258, p=0.0000$, with large effect size [Cohen's $d=1.159$ ]). No significant differences were found in the alpha power of the second HEP component in FP1 $(t[54]=-2.536, p=0.0141$, with large effect size [Cohen's $d=0.690]), \mathrm{FPz}(t[54]=-2.454, p=0.0174$, with large effect size [Cohen's $d=0.668$ ]), or FP2 ( $t$ [54] $=-2.289, p=0.0260$, with large effect size [Cohen's $d$ 

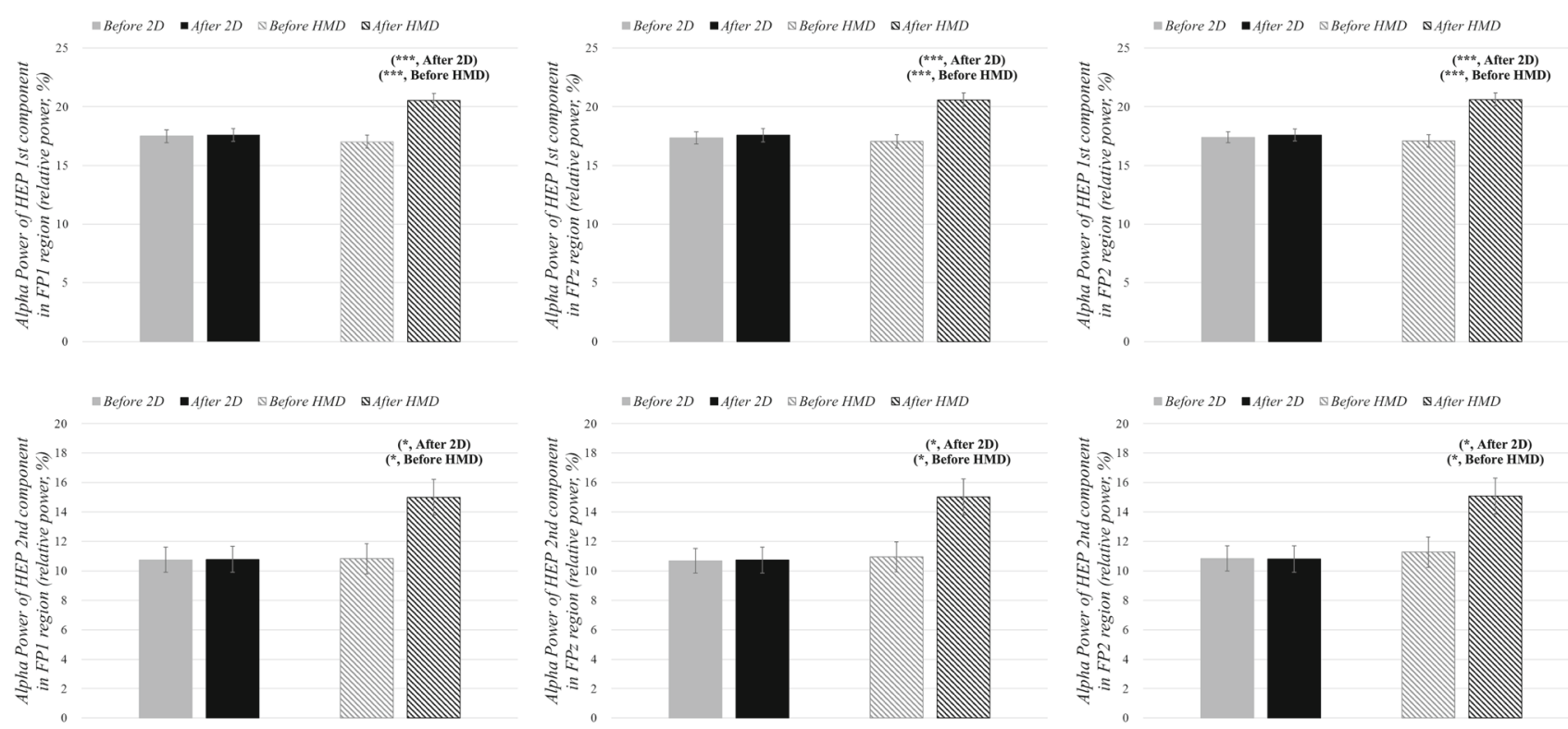

Fig. 7 Average alpha power of first and second HEP components of the $2 \mathrm{D}$ and HMD viewing conditions in the FP1, FPz, and FP2 regions. There was significant difference in the alpha power of the first HEP

component between the 2D and HMD groups in all regions but the alpha power of the second HEP component was not significant based on a paired t-test and ANCOVA $(* p<0.05, * * p<0.0033, * * * p<0.001)$

$=0.623])$. In considering the $2 \mathrm{D}$ viewing tasks, a pairedsamples $t$-test showed that there was no significant difference pre- and post-viewing in either the alpha power of the first or second components in all regions, as shown in Fig. 7.

When comparing the 2D and HMD groups based on ANCOVA, the alpha power of the first HEP component in the HMD group was significantly higher than that in the 2D group in $\mathrm{FP} 1(F[1,54]=151.753, p=0.0001$, with large effect size $\left.\left[\eta_{p}^{2}=0.254\right]\right), \operatorname{FPz}(F[1,54]=154.865, p=$ 0.0001 , with large effect size $\left.\left[\eta_{p}^{2}=0.258\right]\right)$, and FP2 $(F[1$, $54]=149.505, p=0.0001$, with large effect size $\left[\eta_{p}^{2}=\right.$ 0.257]) with the alpha power of the HEP first component adjusted in the pre-viewing condition as a covariate. No significant differences were found in the alpha power of the second HEP component in regions FP1 $(F[1,54]=6.326, p$ $=0.0150$, with medium effect size $\left.\left[\eta_{p}^{2}=0.107\right]\right), \mathrm{FPz}(F[1$, $54]=5.957, p=0.0179$, with medium effect size $\left[\eta_{p}^{2}=\right.$ $0.101])$, and FP2 $(F[1,54]=5.123, p=0.0277$, with medium effect size $\left.\left[\eta_{p}^{2}=0.088\right]\right)$, with the adjusted alpha power of the second HEP component in the pre-viewing condition as a covariate, as shown in Fig. 7.

As an example, one participant's changes in HEP waveform in the FP1, FPz, and FP2 regions before and after viewing the 2D and HMD are shown in Fig. 8. There were minute differences in the dominant positive peak of the first (50-250 ms) and second (250-600 ms) HEP periods before and after viewing in 2D. Interestingly, the dominant peak had an advance after viewing the HMD, instead of before, in both the first and second HEP components. Also, the ampli- tude (difference between the dominant positive and negative peaks) of the HEP waveform before and after viewing in $2 \mathrm{D}$ showed small differences. The amplitude was lower after viewing the HMD content than before watching it.

In analyzing the results of the HMD viewing task, a paired-samples $t$-test showed that the latency of the first HEP component post-viewing was significantly lower than that for the pre-viewing condition in FP1 $(t[54]=5.990, p=$ 0.0000 , with large effect size [Cohen's $d=1.630]$ ), FPz $(t[54]=5.886, p=0.0000$, with large effect size [Cohen's $d=1.602])$, and FP2 $(t[54]=6.342, p=0.0000$, with large effect size [Cohen's $d=1.726]$ ). The latency of the second HEP component post-viewing was also significantly lower compared with the pre-viewing condition in FP1 $(t[54]$ $=4.154, p=0.0001$, with large effect size [Cohen's $d=$ $1.131]), \mathrm{FPz}(t[54]=4.250, p=0.0001$, with large effect size [Cohen's $d=1.157])$, and FP2 $(t[54]=4.317, p=$ 0.0001 , with large effect size [Cohen's $d=1.175]$ ). In analyzing the results of the $2 \mathrm{D}$ viewing tasks, a paired-samples $t$-test showed that there was no significant difference in the latencies of the first and second HEP components pre- and post-viewing in all regions, as shown in Fig. 9.

When comparing the 2D and HMD groups based on ANCOVA, the latency of the first HEP component in the HMD group was significantly lower than that for the 2D group in $\mathrm{FP} 1(F[1,54]=47.625, p=0.0000$, with large effect size $\left.\left[\eta_{p}^{2}=0.473\right]\right), \mathrm{FPz}(F[1,54]=44.355, p=0.0000$, with large effect size $\left.\left[\eta_{p}^{2}=0.456\right]\right)$, and FP2 $(F[1,54]=57.567$, $p=0.0000$, with large effect size $\left.\left[\eta_{p}^{2}=0.521\right]\right)$ with the 

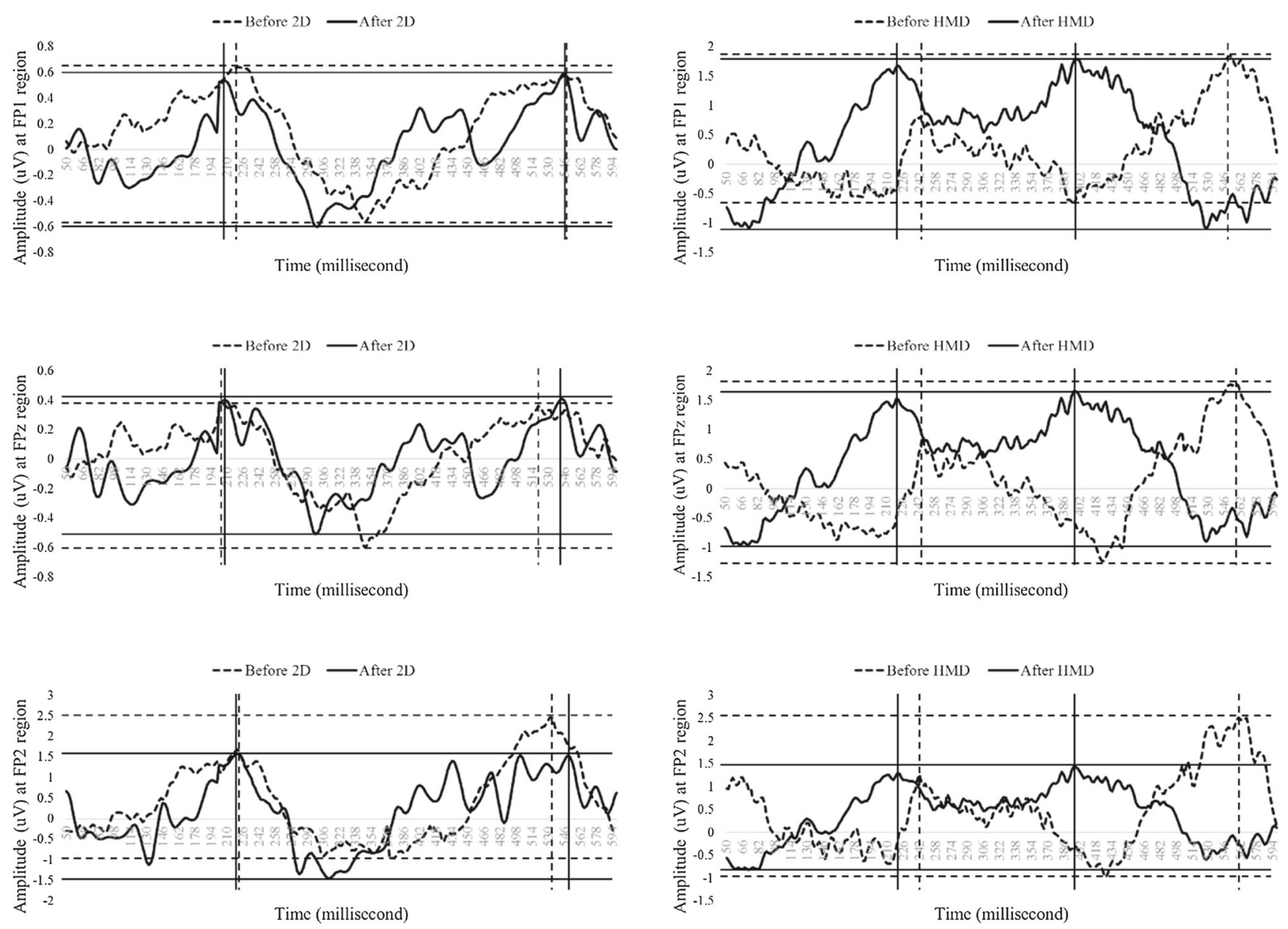

Fig. 8 An example of the changes (before and after viewing) in HEP latency and amplitude for both the 2D and HMD viewing conditions in the FP1, FPz, and FP2 regions for Participant 11. The left and right sides display the results for the 2D and HMD conditions, respectively. The top, mid, and bottom lines are shown FP1, FPz, and FP2 regions, respectively. The difference in HEP latency and amplitude in the FP1, FPz, and FP2 regions are as follows: latency of

the first HEP component (2D: 222-206, 206-208, and 224-222 ms; HMD: 244-220, 246-218, and 242-224 ms), latency of the second HEP component (2D: 548-546, 520-542, and 532-550 ms; HMD: 448-396, 558-390, and 560-394 ms), and amplitude of the HEP (2D: 1.208-1.190, 0.947-0.920, and 3.399-3.223 uV; HMD: 2.907-2.326, $3.226-2.895$, and $3.205-2.721 \mathrm{uV}$ )

adjusted latency of first HEP component in the pre-viewing condition as a covariate. The latency of second HEP component in the HMD group was significantly lower than that for the 2D group in FP1 $(F[1,54]=16.236, p=0.0002$, with large effect size $\left.\left[\eta_{p}^{2}=0.235\right]\right), \operatorname{FPz}(F[1,54]=17.278$, $p=0.0001$, with large effect size $\left.\left[\eta_{p}^{2}=0.246\right]\right)$, and FP2 $\left(F[1,54]=17.919, p=0.0001\right.$, with large effect size $\left[\eta_{p}^{2}\right.$ $=0.253]$ ) with the adjusted latency of second HEP component in the pre-viewing condition as a covariate, as shown in Fig. 9.

In assessing the results of the HMD viewing tasks, a paired-samples $t$-test showed that the amplitude of the HEP post-viewing was significantly lower than that pre-viewing in the $\mathrm{FPz}(t[54]=3.076, p=0.00329$, with large effect size [Cohen's $d=0.837])$ and FP2 $(t[54]=3.557, p=0.0008$,

with large effect size [Cohen's $d=0.968]$ ). No significant differences were found pre- and post-viewing in the amplitudes of the HEP in FP1 $(t[54]=2.639, p=0.0108$, with large effect size [Cohen's $d=0.718]$ ). For the 2D viewing condition, a paired-samples $t$-test showed that there were no significant pre- and post-viewing differences in the latencies of the HEP in all regions, as shown in Fig. 10.

From the comparison between the 2D and HMD groups based on ANCOVA, the amplitude of the HEP in the HMD group was significantly lower than the amplitude measured for the 2D group in FP2 $(F[1,54]=12.475, p=0.0009$, with large effect size $\left.\left[\eta_{p}^{2}=0.191\right]\right)$ with an adjusted amplitude of HEP in the pre-viewing condition as a covariate. However, in the other regions, no significant differences were found in the amplitude of the second HEP component in FP1 ( $F[1$, $54]=6.9976, p=0.0108$, with medium effect size $\left[\eta_{p}^{2}=\right.$ 

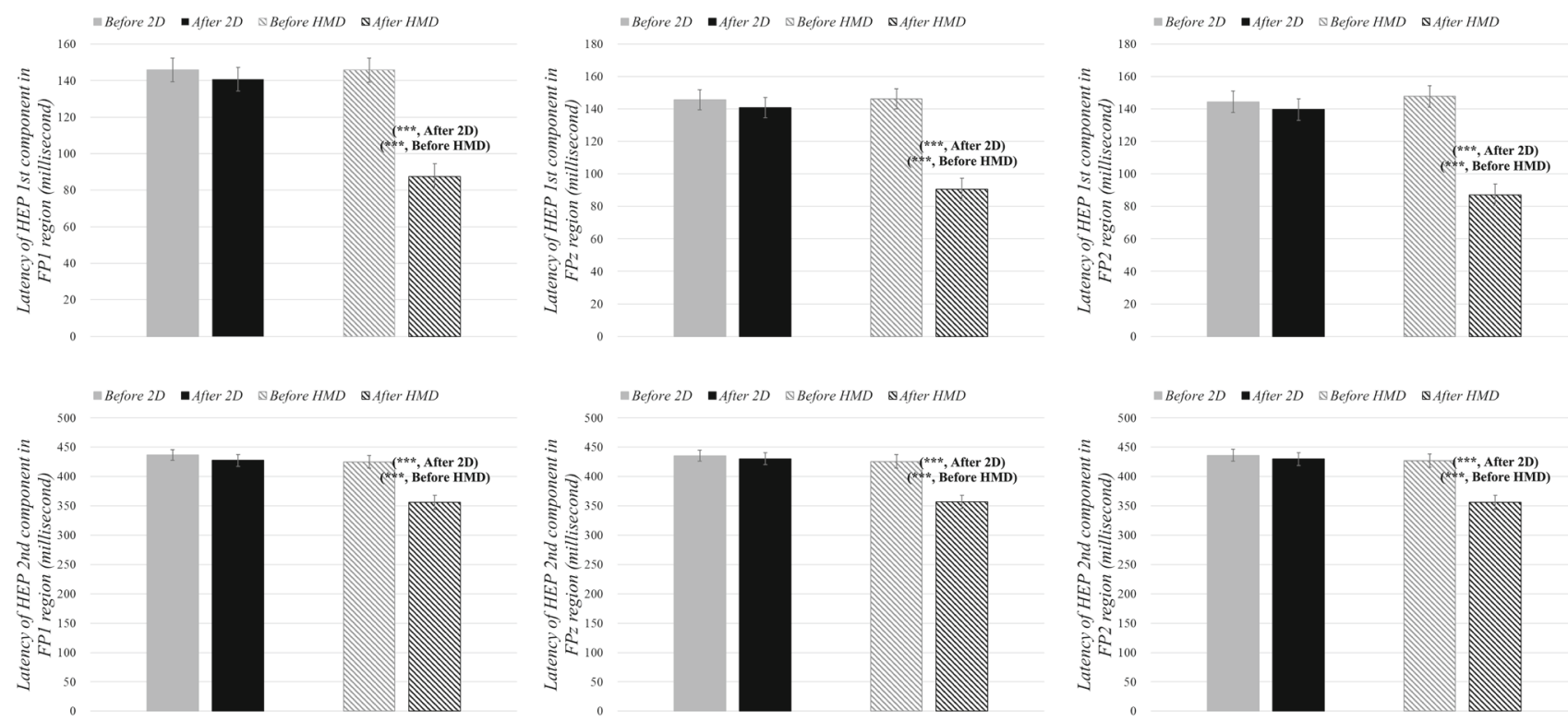

Fig. 9 Average latency of first and second HEP components for the 2D and HMD conditions in the FP1, FPz, and FP2 regions. There was significant difference in the latency of the first and second HEP components

between the 2D and HMD groups in all regions based on a paired t-test and ANCOVA $(* p<0.05, * * p<0.0033, * * * p<0.001)$
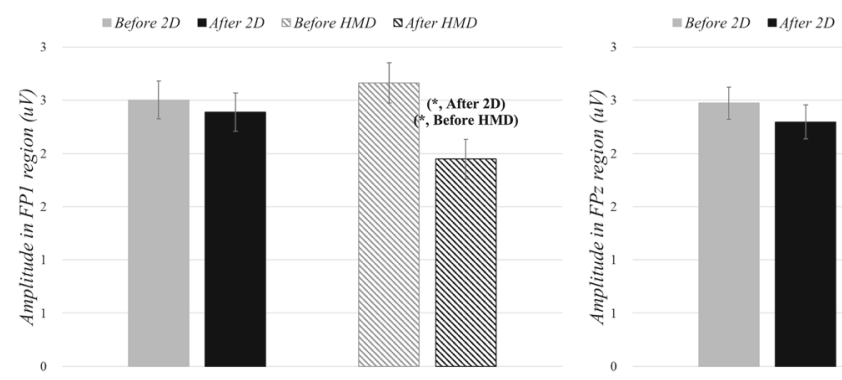

Fig. 10 Average amplitude of the HEP for the 2D and HMD conditions in FP1, FPz, and FP2. There was a significant difference in the amplitude of the HEP between the 2D and HMD groups in FP2 but no significant

$0.116])$ and $\mathrm{FPz}(F[1,54]=9.384, p=0.0034$, with medium effect size $\left.\left[\eta_{p}^{2}=0.150\right]\right)$ with an adjusted alpha power of the second HEP component in the pre-viewing condition as a covariate, as shown in Fig. 10.

\subsection{Correlation analysis}

As seen Fig. 11, we drew the plot for residuals of SSQ scores and significant features of $\operatorname{HEP}\left(\mathrm{AP}(1)_{\mathrm{FP} 1}\right.$, $\mathrm{AP}(1)_{\mathrm{FPz}}, \mathrm{AP}(1)_{\mathrm{FP} 2}, \mathrm{~L}(1)_{\mathrm{FP} 1}, \mathrm{~L}(1)_{\mathrm{FPz}}, \mathrm{L}(1)_{\mathrm{FP} 2}, \mathrm{~L}(2)_{\mathrm{FP} 1}$, $\mathrm{L}(2)_{\mathrm{FPz}}, \mathrm{L}(2)_{\mathrm{FP} 1}, \mathrm{~A}_{\mathrm{FP} 2}$ ) with linear regression lines. Correlation coefficients between SSQ scores and each HEP features in the post-viewing condition considering pre-viewing condition are statistically significant $\left(\mathrm{AP}(1)_{\mathrm{FP} 1}: r=0.531, p\right.$ $<0.05 ; \mathrm{AP}(1)_{\mathrm{FPz}}: r=0.564, p<0.05 ; \mathrm{AP}(1)_{\mathrm{FP} 2}: r=0.542$, $p<0.05 ; L(1)_{\mathrm{FP} 1}: r=0.642, p<0.05 ; \mathrm{L}(1)_{\mathrm{FPz}}: r=0.625, p$

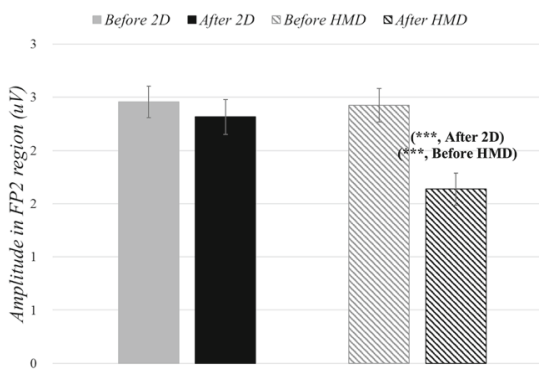

difference in the other regions (FP1 and FPz) based on a paired t-test and ANCOVA $(* p<0.05, * * p<0.0033, * * * p<0.001)$

$<0.05 ; \mathrm{L}(1)_{\mathrm{FP} 2}: r=0.683, p<0.05 ; \mathrm{L}(2)_{\mathrm{FP} 1}: r=0.642, p$ $<0.05 ; \mathrm{L}(2)_{\mathrm{FPz}}: r=0.643, p<0.05 ; \mathrm{L}(2)_{\mathrm{FP} 1}: r=0.628, p$ $\left.<0.05 ; \mathrm{A}_{\mathrm{FP} 2}: r=0.497, p<0.05\right)$.

\subsection{MTMM matrix}

In our research, the multi-method was defined by the 2D and HMD viewing conditions and the multi-trait was defined by measurements such as the alpha power of first HEP component (FP1, FPz, and FP2 regions), the latency of the first and second HEP components (FP1, FPz, and FP2 regions), and the amplitude of HEP (FP2 region) based on measures of statistical significance. The detailed results of the MTMM analysis are shown in Table 2.

Firstly, the test-retest reliability was defined by the main diagonal of the MTMM correlation matrix. The HEP mea- 


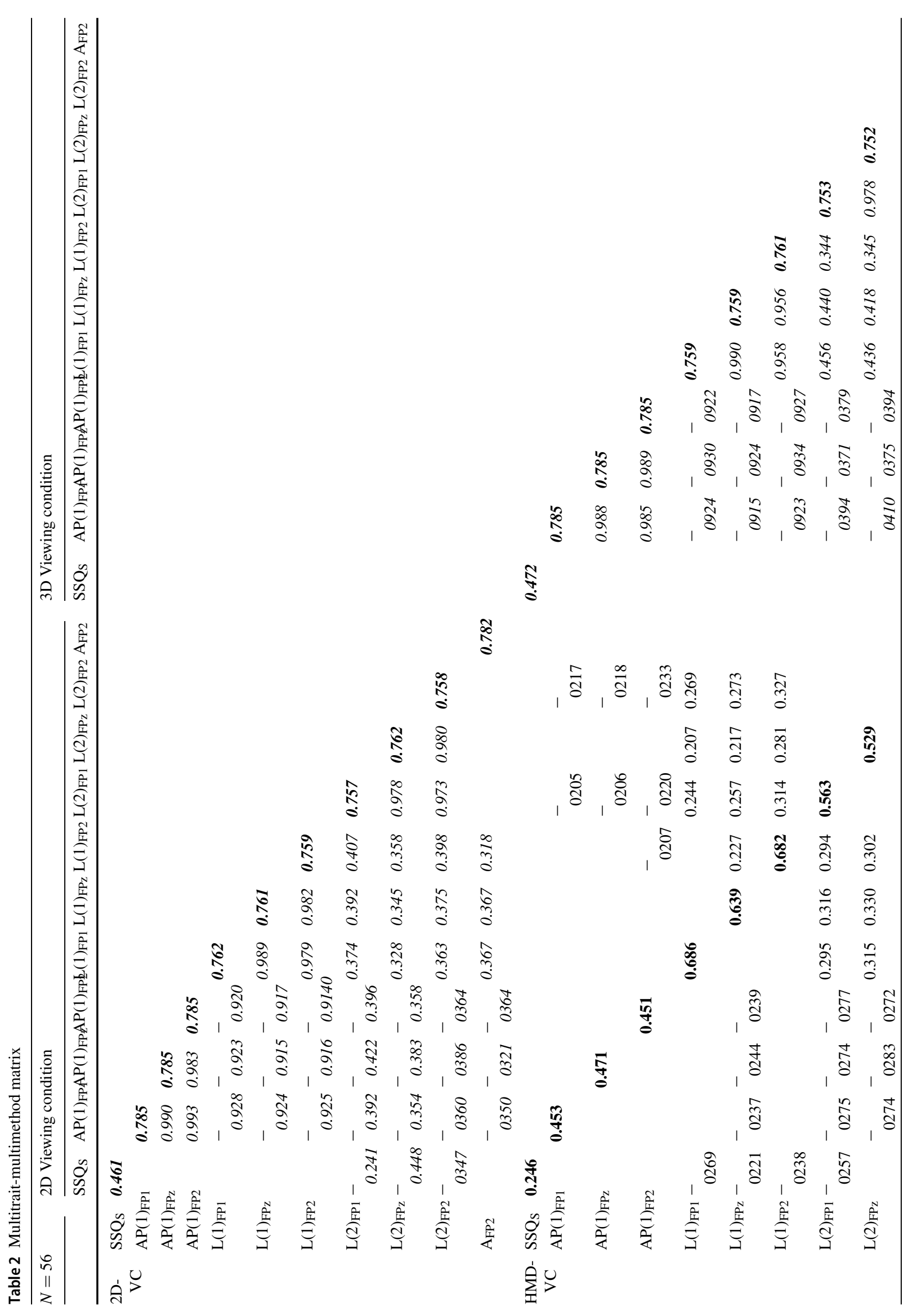




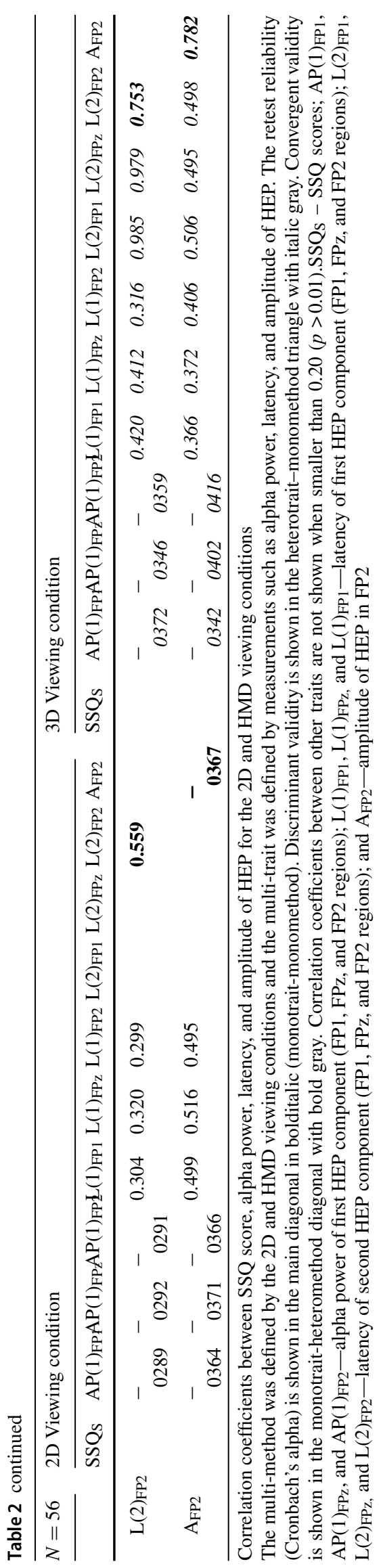



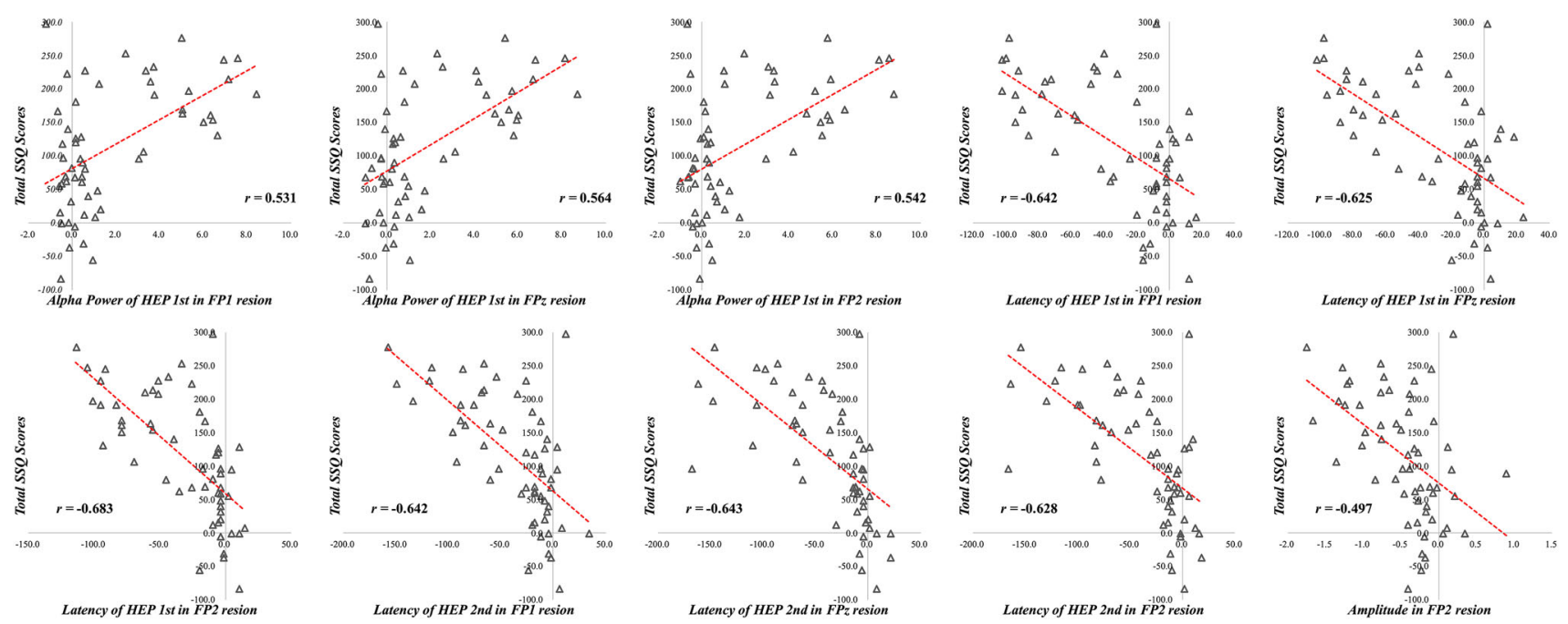

Fig. 11 Results of correlation analysis among HEP features and SSQ score $(p<0.05[n=56])$

sures (alpha power, latency, and amplitude of HEP) showed good reliability in range of 0.752 to 0.785 (over 0.700 ) in both the 2D and HMD viewing conditions. The alpha power of the first HEP component in the FP1, FPz, and FP2 regions revealed good reliability in both the $2 \mathrm{D}(0.785,0.785$, and $0.785)$ and HMD $(0.785,0.785$, and 0.785$)$ viewing conditions. The latency of the first HEP component in FP1, FPz, and FP2 revealed good reliability in both the 2D (first component: $0.761,0.762$, and 0.759 ; second component: 0.757 , 0.762, and 0.758) and HMD (first component: 0.759, 0.790, and 0.761 ; second component: $0.759,0.761$, and 0.753 ) viewing conditions. The amplitude of HEP in FP2 revealed good reliability in both the $2 \mathrm{D}(0.782)$ and HMD (0.782) viewing conditions. The SSQ score showed moderated reliability in both the 2D (0.461) and HMD (0.472) viewing condition, and had low reliability comparison with HEP features. Secondly, the discriminant validity was defined by the heterotraitmonomethod triangles. The correlation coefficients among SSQ score and latency of HEP second component (FP1, FPz, and FP2 regions) showed a low and medium negative correlation ranging from -0.241 to -0.448 , and revealed no significant results with other HEP features. The SSQ score did have discriminant validity with HEP features. The correlation coefficients between the alpha power and latency of the first HEP component measures (FP1, FPz, and FP2 regions) revealed a strong negative correlation in the range of -0.914 to -0.928 . In contrast, the correlation coefficients associated with the latency of the second HEP component demonstrated a medium negative correlation ranging from -0.354 to -0.422 . The correlation coefficients associated with the amplitude of HEP showed a medium negative correlation ranging from -0.321 to -0.416 . The latency of the first HEP component did not have discriminant validity with the alpha power of the first HEP component, but other measures did have discriminant validity with the alpha power measure. Lastly, convergent validity was defined by the monotrait-heteromethod (validity diagonal). The HEP latency measures showed higher correlation (0.529-0.686) than other measures (SSQ score: 0.246; alpha power of first HEP component: 0.451-0.471; amplitude of HEP: - 0.367). In particular, the correlation coefficients for the latency of the first HEP component showed the highest positive correlation (0.639-0.686).

\subsection{Classification}

In this experiment, the motion-sickness and normal groups were classified by linear-SVM, RBF-SVM, elastic net regularization, the LASSO model, and L2 (Ridge model) regularization. Ten features (three alpha powers of the HEP first component in the FP1, FP2, and FPz regions, six latencies of the HEP first and second components in the FP1, FP2, and $\mathrm{FPz}$ regions, and the amplitude of the HEP waveform in FP2 region) were statistically significant for classification (see Figs. 7, 9, and 10). The classification algorithm was trained on the 28 -subject dataset, and its performance was evaluated on a new 20-subject dataset. The comparison results between the 2D and HMD conditions based on the significant features in the 20-subject dataset are shown in Table 3.

The logistic regression classifiers (elastic net regularization and logistic regression with $L 1$ and $L 2$ ) clearly distinguished motion sickness from the normal state, with classification accuracies of $0.850,0.900$, and 0.898 respectively, $F 1$ scores of $0.870,0.870$, and 0.851 respectively, precisions of $0.769,0.769$, and 0.741 respectively, and AUCs of $0.895,0.900$, and 0.898 , respectively. The recall was 1.0 in all three algorithms (Table 4). The regulation parameters 
Table 3 The analysis results of HEP measurements for validation data $(n=20)$ between 2D and HMD conditions

\begin{tabular}{|c|c|c|c|c|c|c|c|c|c|c|c|c|}
\hline & & & \multicolumn{3}{|c|}{ Alpha power } & \multicolumn{5}{|l|}{ Latency } & \multicolumn{2}{|c|}{ Amplitude } \\
\hline & & & $\mathrm{AP}(1)_{\mathrm{FP} 1}$ & $\mathrm{AP}(1)_{\mathrm{FPz}}$ & $\mathrm{AP}(1)_{\mathrm{FP} 2}$ & $\mathrm{~L}(1)_{\mathrm{FP} 1}$ & $\mathrm{~L}(1)_{\mathrm{FPz}}$ & $\mathrm{L}(1)_{\mathrm{FP} 2}$ & $\mathrm{~L}(2)_{\mathrm{FP} 1}$ & $\mathrm{~L}(2)_{\mathrm{FPz}}$ & $\mathrm{L}(2)_{\mathrm{FP} 2}$ & $\mathrm{~A}_{\mathrm{FP} 2}$ \\
\hline \multirow[t]{6}{*}{ 2D-VC } & Before & M & 16.05 & 15.90 & 15.96 & 143.30 & 142.50 & 143.00 & 441.50 & 435.00 & 439.70 & 2.40 \\
\hline & & SD & 2.45 & 2.46 & 2.42 & 34.31 & 33.73 & 31.17 & 60.58 & 61.07 & 62.21 & 0.63 \\
\hline & After & M & 15.29 & 15.19 & 15.24 & 149.10 & 149.70 & 148.40 & 443.50 & 443.80 & 444.70 & 2.52 \\
\hline & & SD & 2.85 & 2.80 & 2.80 & 35.73 & 35.71 & 36.47 & 60.67 & 61.34 & 60.75 & 0.61 \\
\hline & Difference & M & -0.77 & -0.71 & -0.72 & 5.80 & 7.20 & 5.40 & 2.00 & 8.80 & 5.00 & 0.13 \\
\hline & & SD & 1.58 & 1.38 & 1.46 & 16.73 & 13.98 & 14.00 & 10.28 & 17.88 & 14.12 & 0.38 \\
\hline \multirow[t]{6}{*}{ HMD-VC } & Before & $\mathrm{M}$ & 16.05 & 16.20 & 16.12 & 151.20 & 148.50 & 147.80 & 389.10 & 387.50 & 379.90 & 2.35 \\
\hline & & SD & 2.21 & 2.09 & 2.30 & 34.37 & 34.19 & 36.20 & 54.68 & 52.71 & 67.32 & 0.65 \\
\hline & After & M & 19.14 & 19.23 & 18.95 & 116.70 & 116.00 & 116.30 & 347.70 & 346.10 & 350.30 & 1.95 \\
\hline & & SD & 1.44 & 1.35 & 2.24 & 33.19 & 31.91 & 34.58 & 39.33 & 37.98 & 41.98 & 0.53 \\
\hline & Difference & M & 3.09 & 3.03 & 2.83 & -34.50 & -32.50 & -31.50 & -41.40 & -41.40 & -29.60 & -0.39 \\
\hline & & SD & 2.29 & 2.03 & 3.39 & 28.53 & 32.02 & 33.88 & 46.36 & 46.79 & 70.23 & 0.56 \\
\hline
\end{tabular}

$V C$ Viewing condition, $M$ mean, $S D$ standard deviation, $A P(1)_{F P 1}, A P(1)_{F P z}$, and $A P(1)_{F P 2}$ alpha power of first HEP component (FP1, FPz, and FP2 regions); $L(1)_{F P I}, L(1)_{F P z}$ and $L(1)_{F P I}$ latency of first HEP component (FP1, FPz, and FP2 regions); $L(2)_{F P 1}, L(2)_{F P z}$, and $L(2)_{F P I}$ latency of second HEP component (FP1, FPz, and FP2 regions), $A_{F P 2}$ amplitude of HEP in FP2

Table 4 The comparison of the performance of difference types of classifiers

\begin{tabular}{|c|c|c|c|c|c|c|c|c|c|c|}
\hline & \multicolumn{2}{|l|}{ AUC } & \multicolumn{2}{|l|}{ Accuracy } & \multicolumn{2}{|l|}{ F1 Score } & \multicolumn{2}{|l|}{ Precision } & \multicolumn{2}{|l|}{ Recall } \\
\hline & Training & Test & Training & Test & Training & Test & Training & Test & Training & Test \\
\hline $\begin{array}{c}\text { Elastic } \\
\text { Net }\end{array}$ & 0.977 & 0.895 & 0.893 & 0.850 & 0.900 & 0.870 & 0.844 & 0.769 & 0.964 & 1.000 \\
\hline Lasso & 0.950 & 0.900 & 0.929 & 0.850 & 0.933 & 0.870 & 0.875 & 0.769 & 1.000 & 1.000 \\
\hline Ridge & 0.932 & 0.898 & 0.911 & 0.825 & 0.918 & 0.851 & 0.949 & 0.741 & 1.000 & 1.000 \\
\hline $\begin{array}{r}\text { Linear- } \\
\text { SVM }\end{array}$ & 0.992 & 0.898 & 0.946 & 0.850 & 0.949 & 0.870 & 0.903 & 0.769 & 1.000 & 1.000 \\
\hline $\begin{array}{l}\text { RBF- } \\
\text { SVM }\end{array}$ & 0.999 & 0.962 & 0.964 & 0.875 & 0.966 & 0.865 & 0.933 & 0.941 & 1.000 & 0.800 \\
\hline
\end{tabular}
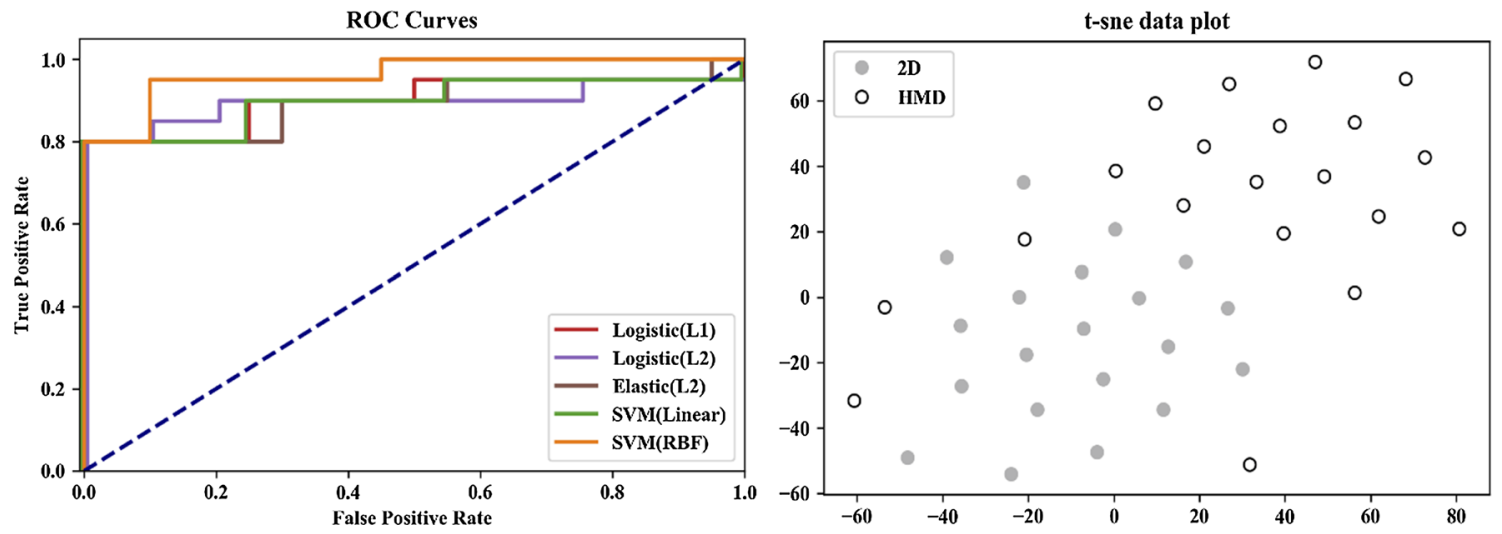

Fig. 12 ROC curves (left) for five classification methods and t-SNE data plot (right)

were $\alpha=4.8$ for Lasso, $\alpha=3.5$ for Ridge, and $\alpha=2.7$ and $\gamma=0.98$ for elastic net. The linear and RBF SVM classifiers also distinguished between motion sickness and the normal state, with classification accuracies of 0.850 and 0.875 respectively, $\mathrm{F} 1$ scores of 0.870 and 0.865 respectively, precisions of 0.769 and 0.941 respectively, recalls of 1.0 and 0.8 respectively, and AUCs of 0.898 and 0.963 , respectively (Table 4). The regulation parameters were $\alpha=1.7$ and $\gamma=$ 9.4 for RBF-SVM, and $\alpha=3.5$ for linear SVM. The ROC curves for evaluating the classification performance, and the 
Table 5 Performance comparison of our method and previous methods in motion-sickness recognition

\begin{tabular}{|c|c|c|c|c|c|c|}
\hline \multirow[t]{2}{*}{ Study } & \multirow[t]{2}{*}{ Subjects } & \multicolumn{2}{|l|}{ Feature extraction } & \multirow[t]{2}{*}{ Classifier } & \multicolumn{2}{|l|}{ Accuracy } \\
\hline & & & & & Training & Test \\
\hline Yu et al. (2010) & 7 & EEG & $\begin{array}{l}\text { PCA, LDA, NWFE, } \\
\text { F/BFS }\end{array}$ & SVM & 0.996 & - \\
\hline Wei et al. (2011) & 6 & EEG & PCA & SVR & 0.869 & - \\
\hline \multirow[t]{2}{*}{ Ko et al. (2013) } & 6 & EEG & e-IBCGA & SVM & 0.807 & - \\
\hline & 6 & & & & - & 0.721 \\
\hline Pane et al. (2018) & 9 & EEG & Beta and theta powers & $\mathrm{CN} 2$ rules & 0.889 & - \\
\hline Mawalid et al. (2018) & 9 & EEG & Alpha and beta powers & Naïve Bayes classifier & 0.838 & - \\
\hline Dennison Jr et al. (2019) & 18 & \multicolumn{2}{|c|}{ Multi-modal (EEG, EOG, RSP, stomach, etc.) } & Tree bagger & 0.950 & - \\
\hline Li et al. (2019) & 20 & EEG \& COP in force plate & PCA & Voting classifier & 0.911 & - \\
\hline Li et al. (2020) & 18 & EEG & WPT & Polynomial-SVM & 0.793 & - \\
\hline Present study & 28 & HEP & Alpha power, amplitude, & RBF-SVM & 0.964 & - \\
\hline & 20 & & and latency of HEP & & - & 0.865 \\
\hline
\end{tabular}

EEG Encephalography, EOG electrooculography; $R S P$ respiration, $C O P$ center of pressure, $H E P$ heartbeat evoked potential, $P C A$ principal component analysis, $L D A$ linear discriminate analysis, $N W F E$ nonparametric weighted feature extraction, $F / B F S$ forward/backward feature selections, $e-I B C G A$ extended bi-objective combinatorial genetic algorithm, WPT wavelet packet transform, $S V M$ support vector machine, $R B F$ radial basis function

t-stochastic neighbor embedding (t-SNE) for vector visualization, are shown in Fig. 12.

\section{Discussion}

VIMS is a major obstacle to the development of the VR industry and the HMD device in particular. Many previous studies have tried to measure the motion sickness in order to resolve this problem. However, the previously proposed methods had limitations and there has not yet been a standardized method suggested. The aim of this study was to develop an advanced method for measuring motion sickness based on cognitive function using heart-brain synchronization by studying HEPs. This study proposed new indicators such as latency and amplitude of HEP to assess motion sickness and compared this with the alpha power of HEP from a previous study based on the MTMM matrix. Based on the SSQ, this study confirmed whether 2D and HMD viewing conditions cause motion sickness. Following the subjective rating obtained from the SSQ, participants experienced motion sickness after the HMD viewing task, but not after the 2D viewing task. This result is consistent with previous studies (Kennedy et al. 1993; Merhi et al. 2007; Sharples et al. 2008; Kiryu et al. 2008; Chardonnet et al. 2015; Palmisano et al. 2017).

Overall, our research yields three significant findings. Firstly, the alpha powers of the first HEP components in the FP1, FPz, and FP2 regions were significantly lower when motion sickness was being experienced. In previous studies, brain sensory processing was found to be influenced by changes in heart rhythm via afferent and efferent pathways, which are related to cognitive functions (Hansen et al. 2003; McCraty et al. 2009; Park et al. 2014, 2015). An increase in the alpha power of the first HEP component is related to the time interval for "rate of change" information to transmit from the heart to the brain through afferent pathways in the vagus nerve (Wölk et al. 1989; McCraty et al. 2009). Park et al. (2015) reported that the alpha power of the first HEP component was increased during cognitive loading and that result is consistent with this research. If information about cardiac rhythm is transmitted rapidly to the brain (increasing the alpha power in the first HEP component), the brain requires information rapidly through sensory input to activate cognitive processing. Thus, as determined by this study, the increasing alpha power of the first HEP component can be interpreted as showing that cognitive load is the cause of motion sickness. Also, many previous studies have demonstrated that motion sickness is strongly related to the cognitive load caused by experiencing VR content (Lin et al. 2007, 2013; Chen et al. 2010; Chuang et al. 2016). An increase in the alpha power of second HEP component is related to the time taken for the pulse wave from the heart to be transmitted to the brain (Wölk et al. 1989; McCraty et al. 2009). If the pulse wave is rapidly transmitted to the brain, the brain requires blood flow to achieve increased information processing. The alpha power of the second HEP component raised during cognitive loading (Park et al. 2015). In the results of this study, the alpha power of the second HEP component in all brain regions tended to decrease during the experience of motion sickness, but this was not statistically significant based on the Bonferroni correction. Secondly, the 
latencies of the first and second HEP components in FP1, $\mathrm{FPz}$, and FP2 were significantly lower during motion sickness. This result also can be interpreted in terms of cognitive load. As mentioned above, the first and second components of the HEP are strongly related to the information transfer rate to the brain from the heart (Wölk et al. 1989; McCraty et al. 2009; Park et al. 2015). Increasing the transfer rate is highly correlated with activating cognitive load, based on the alpha power (Park et al. 2015). Decreasing latencies of first and second HEP components is also related to the information transfer rate to the brain from the heart. The first and second components represent the average time in which cardiac rhythm information is transferred from heart to brain (range 50-250 ms and 250-600 ms after the R-peak) (Wölk et al. 1989; McCraty et al. 2009; Park et al. 2015). Moreover, brain waves in prefrontal and frontal areas are influenced by information about the cardiac rhythm (Schandry et al. 1986; Wölk et al. 1989; Schandry and Weitkunat 1990; McCraty et al. 2009; Park et al. 2015). In this study, the dominant response (peak) from the HEP waveform was extracted from the micro-response in the brain wave caused by the heartbeat using signal averaging techniques. The location of the dominant peak (latency) revealed the time taken for information to be transmitted to the brain from the heart through afferent pathways in the vagus nerve. If the latencies in the first and second HEP components decrease, the increase in the transfer rate from the heart is caused by the requirements of the brain. Thus, decreases in the latency of HEP can be interpreted as cognitive load, thus quantitatively assessing motion sickness. Lastly, the amplitude of the HEP in FP2 was significantly reduced during motion sickness and revealed a medium negative correlation $(-0.321$ to -0.416$)$ with the alpha power of the second HEP component, which was the indicator of cognitive load.

The HEP waveform is the evoked potential caused by the heartbeat in a similar way to the ERP response (by event stimulus). The amplitudes in ERPs are strongly related to high-level cognitive processing measures such as task difficulty, selective attention, and mental workload (Friederici et al. 1993; Uetake and Murata 2000; Kok 2001; Murata et al. 2005; Cheng et al. 2007; Li et al. 2008; Kato et al. 2009; Miller et al. 2011; Mun et al. 2012, 2014; Kathner et al. 2014; Park and Mun 2015; Chang et al. 2017; Getzmann et al. 2018). ERP amplitude is consistent with the inhibition function in the brain. For low level external stimuli, the brain controls decrease the inhibition function to efficiently process the information before revealing a large ERP amplitude (Polich 2007). Thus, decreasing the ERP amplitude is closely related to cognitive load and decreasing the amplitude of the HEP waveform can be interpreted in the same context.

The HEP measures such as alpha power, latency, and amplitude found in this study were significantly different when comparing the HMD (motion sickness inducing) and
$2 \mathrm{D}$ viewing conditions. These measures are strongly related to the cognitive mechanisms underlying the mental workload. Thus, the phenomenon of motion sickness can be interpreted as the degradation of the human visual system due to sensory overload. This is similar to the $3 \mathrm{D}$ visual fatigue found in previous studies (Li et al. 2008; Lambooij et al. 2009; Mun et al. 2012; Park et al. 2014, 2015). The results presented here enable the quantitative assessment of motion sickness and will assist with the establishment of guidelines regarding HMD-based viewing of VR content.

The MTMM matrix, which was found to be very reliable in all HEP measures rather than SSQ score, was internally consistent in both the 2D and HMD conditions. Therefore, the HEP measures showed strongly reliable repeat measurements and consistent and high correlation with the multi-method (2D or HMD). Discriminant validity showed that the alpha power of the first HEP component did not identify a relationship with the latency of the first HEP component but did with other measures such as the latency of the second HEP component and the amplitude of the HEP. The HEP alpha power validated the indicator for cognitive load (Park et al. 2015) and the correlation coefficient between the alpha power and the latency of the first HEP component revealed a strong negative correlation. Generally, electrophysiology features showed high reliability rather than non-electrophysiology such as subjective rating (Park et al. 2015). We found that SSQ score showed significant discrepancy in reliability with electrophysiology measures. Also, the convergent validity (monotrait-heteromethod) was defined by the correlation between two measures of the same trait with two different methods (2D and HMD). Because the two measures are of the same trait, these measures are expected to be strongly correlated. The HEP latency (first component) measures had a higher correlation for the method than other measures. In summary, the latency of the first HEP component had a higher correlation with HEP alpha power, which is well-known as being associated with cognitive load, and higher test-retest reliability and convergent validity than other measures. This measurement, therefore, is recommended to provide a better quantitative evaluation of motion sickness and cognitive load than alpha power and other measures.

Among the algorithms for classifying the motion sickness and normal groups, the RBF-SVM achieved the highest average recognition accuracy ( 0.964 on the training set and 0.875 on the test set). Hence, RBF-SVM is a suitable promising classifier for motion sickness. To better illustrate the study findings, this paper compared the methods and results with those of similar studies. In previous studies, the accuracy of recognizing motion sickness was $0.793-0.996$ in the training set and 0.721 in the test set (one example), as shown in Table. 5. In terms of accuracy, sample size, and validation results, 
our methods outperformed the existing state-of-the-art classification methods for motion-sickness detection.

\section{Conclusion}

The aim of this study was to determine a method for measuring motion sickness experience by watching VR content on a HMD using the HEP phenomenon and to propose a new indicator for evaluating motion sickness (cognitive function). This study confirmed that motion sickness leads to a decay in cognitive processing in the brain caused by multi-sensory input as demonstrated by reductions in the alpha power of the first HEP component in regions FP1, FPz, and FP2. Also, the proposed indicators such as latency (first component in FP1, FPz, and FP2) and amplitude (FP2) of the HEP waveform in this study were significantly different when participants experienced motion sickness and showed higher correlations with alpha power measures (cognitive load). In particular, latencies in the first HEP component was recommended as better quantitative evaluators of motion sickness (cognitive load) than alpha power and other measures when test-retest reliability, discriminant, and convergent validity were verified by the MTMM matrix. Because the HEP measures were extracted from the HEP waveforms of the heartbeat, the proposed method is more flexible than offline methods such as the ERP method, which requires specific tasks. In addition, our proposed method implemented in RBF-SVM more successfully classified the motion-sickness state than state-of-the-art recognition methods for motion sickness, demonstrating a higher performance than previous studies. The proposed HEP measurement method is useful for quantifying motion sickness and determining the optimal viewing parameters of VR content, including the viewer characteristics, viewing environment, content, and device factors. These results will improve the popularization of VR and invigorate the development of future VR with suppressed negative side effects.

Authors' contributions Sangin Park contributed to conceptualization, methodology, writing-original draft, Soo Ji Choi contributed to investigation, visualization, software, data curation, Laehyun Kim contributed to conceptualization, writing-review \& editing, and Mincheol Whang contributed to supervision, writing — review \& editing.

Funding This work was supported by the Electronics and Telecommunications Research Institute (ETRI) grant funded by the Korean government [21ZS1100, Core Technology Research for Self-Improving Integrated Artificial Intelligence System] and Institute of Information and communications Technology Planning \& Evaluation (IITP) grant funded by the Korea government (MSIT) (No. 2017-0-00432, Development of non-invasive integrated BCI SW platform to control home appliances and external devices by user's thought via AR/VR interface).

Availability of data and material Not applicable.

\section{Declarations}

Ethics approval Not applicable.

Consent to participate Not applicable.

Consent for publication Not applicable.

Conflicts of interest These authors declare that they have no conflict of interest.

Open Access This article is licensed under a Creative Commons Attribution 4.0 International License, which permits use, sharing, adaptation, distribution and reproduction in any medium or format, as long as you give appropriate credit to the original author(s) and the source, provide a link to the Creative Commons licence, and indicate if changes were made. The images or other third party material in this article are included in the article's Creative Commons licence, unless indicated otherwise in a credit line to the material. If material is not included in the article's Creative Commons licence and your intended use is not permitted by statutory regulation or exceeds the permitted use, you will need to obtain permission directly from the copyright holder. To view a copy of this licence, visit http://creativecomm ons.org/licenses/by/4.0/.

\section{References}

Annett J (2002) Subjective rating scales: science or art? Ergon 45:966-987. https://doi.org/10.1080/00140130210166951

Azuma RT (1997) A survey of augmented reality. Presence: Teleoper Virtual Environ 6:355-385

Bailenson J, Patel K, Nielsen A, Bajscy R, Jung S-H, Kurillo G (2008) The effect of interactivity on learning physical actions in virtual reality. Media Psychol 11:354-376. https://doi.org/10.1080/ 15213260802285214

Bos JE, Bles W, Groen EL (2008) A theory on visually induced motion sickness. Displays 29:47-57. https://doi.org/10.1016/j. displa.2007.09.002

Bos JE, de Vries SC, van Emmerik ML, Groen EL (2010) The effect of internal and external fields of view on visually induced motion sickness. Appl Ergon 41:516-521. https://doi.org/10.1016/ j.apergo.2009.11.007

Bouchard S, Robillard G, Renaud P, Bernier F (2011) Exploring new dimensions in the assessment of virtual reality induced side effects. J Comput Inf Technol 1:20-32

Boussaoud D (2001) Attention versus intention in the primate premotor cortex. Neuroimage 14:S40-45. https://doi.org/10.1006/nimg. 2001.0816

Cain B (2007) A review of the mental workload literature. Defence Research And Development, Toronto

Campbell DT, Fiske DW (1959) Convergent and discriminant validation by the multitrait-multimethod matrix. Psychol Bull 56:81-105. https://doi.org/10.1037/h0046016

Carnegie K, Rhee T (2015) Reducing visual discomfort with HMDs using dynamic depth of field. IEEE Comput Graph Appl 35:34-41. https://doi.org/10.1109/MCG.2015.98

Cessie SL, Houwelingen JCV (1992) Ridge estimators in logistic regression. Appl Stat 41:191-201. https://doi.org/10.2307/2347628

Chang Y-W, Hsieh C-J, Chang K-W, Ringgaard M, Lin C-J (2010) Training and testing low-degree polynomial data mappings via linear SVM. J Mach Learn Res 11:1471-1490

Chang YK, Alderman BL, Chu CH, Wang CC, Song TF, Chen FT (2017) Acute exercise has a general facilitative effect on 
cognitive function: a combined ERP temporal dynamics and BDNF study. Psychophysiology 54:289-300. https://doi.org/10. 1111/psyp. 12784

Chardonnet J-R, Mirzaei MA, Merienne F (2015) Visually induced motion sickness estimation and prediction in virtual reality using frequency components analysis of postural sway signal. In: International conference on artificial reality and telexistence eurographics symposium on virtual environments, pp 9-16

Chen YC, Duann JR, Chuang SW et al (2010) Spatial and temporal EEG dynamics of motion sickness. Neuroimage 49:2862-2870. https://doi.org/10.1016/j.neuroimage.2009.10.005

Cheng S, Lee H, Shu C, Hsu H (2007) Electroencephalographic study of mental fatigue in visual display terminal tasks. J Med Biol Eng 27:124-131

Chuang SW, Chuang CH, Yu YH, King JT, Lin CT (2016) EEG alpha and gamma modulators mediate motion sickness-related spectral responses. Int J Neural Syst 26:1650007. https://doi.org/10.1142/ S0129065716500076

Clemente M, Rodríguez A, Rey B, Alcañiz M (2014) Assessment of the influence of navigation control and screen size on the sense of presence in virtual reality using EEG. Expert Syst Appl 41:1584-1592. https://doi.org/10.1016/j.eswa.2013.08.055

Davis AM, Natelson BH (1993) Brain-heart interactions. Brain-heart interactions. The neurocardiology of arrhythmia and sudden cardiac death. Tex Heart Inst J 20:158-169

de Morree HM, Klein C, Marcora SM (2014) Cortical substrates of the effects of caffeine and time-on-task on perception of effort. J Appl Physiol 117:1514-1523. https://doi.org/10.1152/ japplphysiol.00898.2013

Diels C, Ukai K, Howarth PA (2007) Visually induced motion sickness with radial displays: effects of gaze angle and fixation. Aviat Space Environ Med 78:659-665

Diykh M, Li Y (2016) Complex networks approach for EEG signal sleep stages classification. Expert Syst Appl 63:241-248. https:// doi.org/10.1016/j.eswa.2016.07.004

Drew RC, Bell MP, White MJ (2008) Modulation of spontaneous baroreflex control of heart rate and indexes of vagal tone by passive calf muscle stretch during graded metaboreflex activation in humans. J Appl Physiol 104:716-723. https://doi.org/10.1152/ japplphysiol.00956.2007

Dunnett CW (1955) A Multiple Comparison procedure for comparing several treatments with a control. J Am Stat Assoc 50:1096-1121. https://doi.org/10.2307/2281208

Friederici AD, Pfeifer E, Hahne A (1993) Event-related brain potentials during natural speech processing: effects of semantic, morphological and syntactic violations. Brain Res Cogn Brain Res 1:183-192. https://doi.org/10.1016/0926-6410(93)90026-2

Fukushima H, Terasawa Y, Umeda S (2011) Association between interoception and empathy: evidence from heartbeat-evoked brain potential. Int J Psychophysiol 79:259-265. https://doi.org/10. 1016/j.ijpsycho.2010.10.015

Fuster JM (1988) Prefrontal cortex. In: Comparative neuroscience and neurobiology. Springer, Birkhäuser, Boston

Getzmann S, Wascher E, Schneider D (2018) The role of inhibition for working memory processes: ERP evidence from a short-term storage task. Psychophysiology 55:e13026. https://doi.org/10.1111/ psyp. 13026

Gianaros PJ, Quigley KS, Muth ER, Levine ME, JrRC V, Stern RM (2003) Relationship between temporal changes in cardiac parasympathetic activity and motion sickness severity. Psychophysiology 40:39-44. https://doi.org/10.1111/1469-8986. 00005

Hansen AL, Johnsen BH, Thayer JF (2003) Vagal influence on working memory and attention. Int J Psychophysiol 48:263-274. https:// doi.org/10.1016/s0167-8760(03)00073-4
Hartikainen KM, Knight RT (2003) Lateral and orbital prefrontal cortex contributions to attention. In: Detection of change. Springer, Boston

Herrera LJ, Fernandes CM, Mora AM et al (2013) Combination of heterogeneous EEG feature extraction methods and stacked sequential learning for sleep stage classification. Int J Neural Syst 23:1350012. https://doi.org/10.1142/S0129065713500123

Hettinger LJ, Riccio GE (1992) Visually induced motion sickness in virtual environments. Presence: Teleoper Virt Environ. 1:306-310. https://doi.org/10.1162/pres.1992.1.3.306

Höllerer T, Feiner S, Terauchi T, Rashid G, Hallaway D (1999) Exploring MARS: developing indoor and outdoor user interfaces to a mobile augmented reality system. Comput Graph 23:779-785. https://doi.org/10.1016/S0097-8493(99)00103-X

Huck SW, Cormier WH, Bounds WG (1974) Reading statistics and research. Harper \& Row, New York

James G, Witten D, Hastie T, Tibshirani R (2013) An introduction to statistical learning. Springer, New York

Janig W (1996) Neurobiology of visceral afferent neurons: neuroanatomy, functions, organ regulations and sensations. Biol Psychol 42:29-51. https://doi.org/10.1016/0301-0511(95)051457

Kathner I, Wriessnegger SC, Muller-Putz GR, Kubler A, Halder S (2014) Effects of mental workload and fatigue on the P300, alpha and theta band power during operation of an ERP (P300) braincomputer interface. Biol Psychol 102:118-129. https://doi.org/10. 1016/j.biopsycho.2014.07.014

Kato Y, Endo H, Kizuka T (2009) Mental fatigue and impaired response processes: event-related brain potentials in a Go/NoGo task. Int J Psychophysiol 72:204-211. https://doi.org/10.1016/j.ijpsycho. 2008.12.008

Kennedy RS, Drexler J, Kennedy RC (2010) Research in visually induced motion sickness. Appl Ergon 41:494-503. https://doi.org/ 10.1016/j.apergo.2009.11.006

Kennedy RS, Lane NE, Berbaum KS, Lilienthal MG (1993) Simulator sickness questionnaire: an enhanced method for quantifying simulator sickness. Int J Aviat Psychol 3:203-220. https://doi.org/10. 1207/s15327108ijap0303_3

Keselman HJ, Huberty CJ, Lix LM et al (2016) Statistical practices of educational researchers: an analysis of their ANOVA, MANOVA, and ANCOVA analyses. Rev Educ Res 68:350-386. https://doi. org/10.3102/00346543068003350

Kesim M, Ozarslan Y (2012) Augmented reality in education: current technologies and the potential for education. Procedia Soc Behav Sci 47:297-302. https://doi.org/10.1016/j.sbspro.2012.06.654

Kim S, Lee S, Kala N, Lee J, Choe W (2018) An effective FoV restriction approach to mitigate VR sickness on mobile devices. J Soc Inf Disp 26:376-384. https://doi.org/10.1002/jsid.669

Kim YY, Kim HJ, Kim EN, Ko HD, Kim HT (2005) Characteristic changes in the physiological components of cybersickness. Psychophysiology 425:616-625. https://doi.org/10.1111/j.14698986.2005.00349.x

Kiryu T, Tada G, Toyama H, Iijima A (2008) Integrated evaluation of visually induced motion sickness in terms of autonomic nervous regulation. In: 2008 30th annual international conference of the ieee engineering in medicine and biology society, pp 4597-4600. https://doi.org/10.1109/IEMBS.2008.4650237

Kok A (2001) On the utility of P3 amplitude as a measure of processing capacity. Psychophysiol 38:557-577. https://doi.org/10.1017/ s0048577201990559

Lambooij M, Ijsselsteijn W, Fortuin M, Heynderickx I (2009) Visual discomfort and visual fatigue of stereoscopic displays: a review. J Imaging Technol 53:30201-30201-30201-30214. https://doi.org/ 10.2352/J.ImagingSci.Technol.2009.53.3.030201

Lambooij MT, IJsselsteijn WA, Heynderickx I (2007) Visual discomfort in stereoscopic displays: a review. In: Stereoscopic displays and 
virtual reality systems XIV, international society for optics and photonics, vol 6490, p 64900I. https://doi.org/10.1117/12.705527

Lechinger J, Heib DP, Gruber W, Schabus M, Klimesch W (2015) Heartbeat-related EEG amplitude and phase modulations from wakefulness to deep sleep: interactions with sleep spindles and slow oscillations. Psychophysiology 52:1441-1450. https://doi. org/10.1111/psyp. 12508

Li H-CO, Seo J, Kham K, Lee S (2008) Measurement of 3D visual fatigue using event-related potential (ERP): $3 \mathrm{D}$ oddball paradigm. In 2008 3DTV conference: the true vision-capture, transmission and display of 3D video, pp 213-216. https://doi.org/10.1109/ 3DTV.2008.4547846

Li W, Liu H, Yang P, Xie W (2016) Supporting regularized logistic regression privately and efficiently. PLoS ONE 11:e0156479. https://doi.org/10.1371/journal.pone.0156479

Lima CAM, Coelho ALV, Chagas S (2009) Automatic EEG signal classification for epilepsy diagnosis with relevance vector machines. Expert Syst Appl 36:10054-10059. https://doi.org/10. 1016/j.eswa.2009.01.022

Lin C-T, Chuang S-W, Chen Y-C, Ko L-W, Liang S-F, Jung T-P (2007) EEG effects of motion sickness induced in a dynamic virtual reality environment. In: 2007 29th annual international conference of the IEEE engineering in medicine and biology society, pp 3872-3875. https://doi.org/10.1109/IEMBS.2007.4353178

Lin CT, Tsai SF, Ko LW (2013) EEG-based learning system for online motion sickness level estimation in a dynamic vehicle environment. IEEE Trans Neural Netw Learn Syst 24:1689-1700. https:// doi.org/10.1109/TNNLS.2013.2275003

Malinska M, Zuzewicz K, Bugajska J, Grabowski A (2015) Heart rate variability (HRV) during virtual reality immersion. Int $\mathbf{J}$ Occup Saf Ergon 21:47-54. https://doi.org/10.1080/10803548. 2015.1017964

Mazloumi Gavgani A, Walker FR, Hodgson DM, Nalivaiko E (2018) A comparative study of cybersickness during exposure to virtual reality and "classic" motion sickness: are they different? J Appl Physiol 125:1670-1680. https://doi.org/10.1152/japplphysiol.00338.2018

McCraty R, Atkinson M, Tomasino D, Bradley RT (2009) The coherent heart heart-brain interactions, psychophysiological coherence, and the emergence of system-wide order. Integral Rev: A Transdiscipl Transcult J New Thought, Res, Praxis 5:10-115

McGibbon CA, Krebs DE (2004) Discriminating age and disability effects in locomotion: neuromuscular adaptations in musculoskeletal pathology. J Appl Physiol 96:149-160. https://doi.org/ 10.1152/japplphysiol.00422.2003

Merhi O, Faugloire E, Flanagan M, Stoffregen TA (2007) Motion sickness, console video games, and head-mounted displays. Hum Factors 49:920-934. https://doi.org/10.1518/001872007X230262

Miller MW, Rietschel JC, McDonald CG, Hatfield BD (2011) A novel approach to the physiological measurement of mental workload. Int J Psychophysiol 80:75-78. https://doi.org/10.1016/j.ijpsycho. 2011.02.003

Mon-Williams M, Wann JP, Rushton S (1993) Binocular vision in a virtual world: visual deficits following the wearing of a head-mounted display. Ophthalmic Physiol Opt 13:387-391. https://doi.org/10. 1111/j.1475-1313.1993.tb00496.x

Montoya P, Schandry R, Muller A (1993) Heartbeat evoked potentials (HEP): topography and influence of cardiac awareness and focus of attention. Electroencephalogr Clin Neurophysiol 88:163-172. https://doi.org/10.1016/0168-5597(93)90001-6

Morris NB, Bain AR, Cramer MN, Jay O (2014) Evidence that transient changes in sudomotor output with cold and warm fluid ingestion are independently modulated by abdominal, but not oral thermoreceptors. J Appl Physiol 116:1088-1095. https://doi.org/10.1152/ japplphysiol.01059.2013
Moss JD, Muth ER (2011) Characteristics of head-mounted displays and their effects on simulator sickness. Hum Factors 53:308-319. https://doi.org/10.1177/0018720811405196

Mullen T, Kothe C, Chi YM et al. Real-time modeling and 3D visualization of source dynamics and connectivity using wearable EEG. In: 2013 35th annual international conference of the IEEE engineering in medicine and biology society (EMBC), pp 2184-2187. https://doi.org/10.1109/EMBC.2013.6609968

Mun S, Kim ES, Park MC (2014) Effect of mental fatigue caused by mobile 3D viewing on selective attention: an ERP study. Int J Psychophysiol 94:373-381. https://doi.org/10.1016/j.ijpsycho.2014. 08.1389

Mun S, Park MC, Park S, Whang M (2012) SSVEP and ERP measurement of cognitive fatigue caused by stereoscopic 3D. Neurosci Lett 525:89-94. https://doi.org/10.1016/j.neulet.2012.07.049

Murata A, Uetake A, Takasawa Y (2005) Evaluation of mental fatigue using feature parameter extracted from event-related potential. Int J Ind Ergon 35:761-770. https://doi.org/10.1016/j.ergon.2004.12. 003

Nalivaiko E, Davis SL, Blackmore KL, Vakulin A, Nesbitt KV (2015) Cybersickness provoked by head-mounted display affects cutaneous vascular tone, heart rate and reaction time. Physiol Behav 151:583-590. https://doi.org/10.1016/j.physbeh.2015.08.043

Naqvi SAA, Badruddin N, Malik AS, Hazabbah W, Abdullah B (2013) Does 3D produce more symptoms of visually induced motion sickness? In: 2013 35th annual international conference of the IEEE engineering in medicine and biology society (EMBC), pp 6405-6408

Nauta WJ, Feirtag M, Donner C (1986) Fundamental neuroanatomy. Freeman, New York

Nieuwenhuys R, Voogd J, Van Huijzen C (2007) The human central nervous system: a synopsis and atlas. Springer, Berlin

Ohyama S, Nishiike S, Watanabe $\mathrm{H}$ et al (2007) Autonomic responses during motion sickness induced by virtual reality. Auris Nasus Larynx 34:303-306. https://doi.org/10.1016/j.anl.2007.01.002

Palmisano S, Mursic R, Kim J (2017) Vection and cybersickness generated by head-and-display motion in the Oculus Rift. Displays 46:1-8. https://doi.org/10.1016/j.displa.2016.11.001

Pan J, Tompkins WJ (1985) A real-time QRS detection algorithm. IEEE Trans Biomed Eng 32:230-236. https://doi.org/10.1109/TBME. 1985.325532

Pan Z, Cheok AD, Yang H, Zhu J, Shi J (2006) Virtual reality and mixed reality for virtual learning environments. Comput Graph 30:20-28. https://doi.org/10.1016/j.cag.2005.10.004

Park M-C, Mun S (2015) Overview of measurement methods for factors affecting the human visual system in 3D displays. J Disp Technol 11:877-888. https://doi.org/10.1109/jdt.2015.2389212

Park S, Mun S, Lee DW, Whang M (2019) IR-camera-based measurements of 2D/3D cognitive fatigue in 2D/3D display system using task-evoked pupillary response. Appl Opt 58:3467-3480. https:// doi.org/10.1364/AO.58.003467

Park S, Won MJ, Lee EC, Mun S, Park MC, Whang M (2015) Evaluation of 3D cognitive fatigue using heart-brain synchronization. Int J Psychophysiol 97:120-130. https://doi.org/10.1016/j.ijpsycho. 2015.04.006

Park S, Won MJ, Mun S, Lee EC, Whang M (2014) Does visual fatigue from 3D displays affect autonomic regulation and heart rhythm? Int J Psychophysiol 92:42-48. https://doi.org/10.1016/j.ijpsycho. 2014.02.003

Pereira JM, Basto M, Silva AFD (2016) The logistic lasso and ridge regression in predicting corporate failure. Procedia Econ Finance 39:634-641. https://doi.org/10.1016/s2212-5671(16)30310-0

Polich J (2007) Updating P300: an integrative theory of P3a and P3b. Clin Neurophysiol 118:2128-2148. https://doi.org/10.1016/ j.clinph.2007.04.019 
Pollatos O, Schandry R (2004) Accuracy of heartbeat perception is reflected in the amplitude of the heartbeat-evoked brain potential. Psychophysiology 41:476-482. https://doi.org/10.1111/14698986.2004.00170.x

Porges SW (1997) Emotion: an evolutionary by-product of the neural regulation of the autonomic nervous system. Ann N Y Acad Sci 807:62-77. https://doi.org/10.1111/j.1749-6632.1997.tb51913.x

Porges SW (2007) The polyvagal perspective. Biol Psychol 74:116-143. https://doi.org/10.1016/j.biopsycho.2006.06.009

Psotka J (1995) Immersive training systems: virtual reality and education and training. Instr Sci 23:405-431. https://doi.org/10.1007/ bf00896880

Raajan NR, Suganya S, Priya MV et al (2012) Augmented reality based virtual reality. Procedia Eng 38:1559-1565. https://doi.org/ 10.1016/j.proeng.2012.06.191

Regan C (1995) An investigation into nausea and other side-effects of head-coupled immersive virtual reality. Virtual Real 1(1):17-31. https://doi.org/10.1007/BF02009710

Regan EC, Ramsey AD (1996) The efficacy of hyoscine hydrobromide in reducing side-effects induced during immersion in virtual reality. Aviat Space Environ Med 67:222-226

Rodríguez A, Rey B, Clemente M, Wrzesien M, Alcañiz M (2015) Assessing brain activations associated with emotional regulation during virtual reality mood induction procedures. Expert Syst Appl 42:1699-1709. https://doi.org/10.1016/j.eswa.2014.10.006

Ryan M-L (1999) Immersion vs. interactivity: virtual reality and literary theory. SubStance 28:110-137. https://doi.org/10.1353/sub.1999. 0015

Saito T, Rehmsmeier M (2017) Precrec: fast and accurate precisionrecall and ROC curve calculations in R. Bioinform 33:145-147. https://doi.org/10.1093/bioinformatics/btw570

Schandry R, Montoya P (1996) Event-related brain potentials and the processing of cardiac activity. Biol Psychol 42:75-85. https://doi. org/10.1016/0301-0511(95)05147-3

Schandry R, Sparrer B, Weitkunat R (1986) From the heart to the brain: a study of heartbeat contingent scalp potentials. Int J Neurosci 30:261-275. https://doi.org/10.3109/00207458608985677

Schandry R, Weitkunat R (1990) Enhancement of heartbeat-related brain potentials through cardiac awareness training. Int J Neurosci 53:243-253. https://doi.org/10.3109/00207459008986611

Sharples S, Cobb S, Moody A, Wilson JR (2008) Virtual reality induced symptoms and effects (VRISE): comparison of head mounted display (HMD), desktop and projection display systems. Displays 29:58-69. https://doi.org/10.1016/j.displa.2007.09.005

Smart LJ, Stoffregen TA, Bardy BG (2002) Visually induced motion sickness predicted by postural instability. Hum Factors 44:451-465. https://doi.org/10.1518/0018720024497745

Steuer J (1992) Defining virtual reality: dimensions determining telepresence. J Commun 42:73-93. https://doi.org/10.1111/j.14602466.1992.tb00812.x

Stolz C, Endres D, Mueller EM (2019) Threat-conditioned contexts modulate the late positive potential to faces-A mobile EEG/virtual reality study. Psychophysiol 56:e13308. https://doi.org/10.1111/ psyp. 13308

Uetake A, Murata A (2000) Assessment of mental fatigue during VDT task using event-related potential (P300). In: Proceedings 9th IEEE international workshop on robot and human interactive communication, pp 235-240. https://doi.org/10.1109/ROMAN.2000. 892501
Uijtdehaage SH, Stern RM, Koch KL (1992) Effects of eating on vection-induced motion sickness, cardiac vagal tone, and gastric myoelectric activity. Psychophysiology 29:193-201. https://doi. org/10.1111/j.1469-8986.1992.tb01685.x

Van Krevelen DWF, Poelman R (2010) A Survey of augmented reality technologies, applications and limitations. Int J Virtual Real 9:1-20. https://doi.org/10.20870/ijvr.2010.9.2.2767

Villena-Gonzalez M, Moenne-Loccoz C, Lagos RA et al (2017) Attending to the heart is associated with posterior alpha band increase and a reduction in sensitivity to concurrent visual stimuli. Psychophysiol 54:1483-1497. https://doi.org/10.1111/psyp.12894

Warner HR, Cox A (1962) A mathematical model of heart rate control by sympathetic and vagus efferent information. J Appl Physiol 17:349-355. https://doi.org/10.1152/jappl.1962.17.2.349

Wölk C, Velden M, Zimmermann U, Krug S (1989) The interrelation between phasic blood pressure and heart rate changes in the context of the" baroreceptor hypothesis. J Psychophysiol 3:397-402

Yokota Y, Aoki M, Mizuta K, Ito Y, Isu N (2005) Motion sickness susceptibility associated with visually induced postural instability and cardiac autonomic responses in healthy subjects. Acta Otolaryngol 125:280-285. https://doi.org/10.1080/00016480510003192

Zhang Y, Jin J, Qing X, Wang B, Wang X (2012) LASSO based stimulus frequency recognition model for SSVEP BCIs. Biomed Signal Proces 7:104-111. https://doi.org/10.1016/j.bspc.2011.02.002

Zhao C, Zheng C, Zhao M, Tu Y, Liu J (2011) Multivariate autoregressive models and kernel learning algorithms for classifying driving mental fatigue based on electroencephalographic. Expert Syst Appl 38:1859-1865. https://doi.org/10.1016/j.eswa.2010.07. 115

Zhou T, Tao D, Wu X (2010) Manifold elastic net: a unified framework for sparse dimension reduction. Data Min Knowl Discov 22:340-371. https://doi.org/10.1007/s10618-010-0182-x

Zou H, Hastie T (2005) Regularization and variable selection via the elastic net. J R Stat Soc Series B 67:301-320. https://doi.org/10. 1111/j.1467-9868.2005.00503.x

Zuzewicz K, Saulewicz A, Konarska M, Kaczorowski Z (2011) Heart rate variability and motion sickness during forklift simulator driving. Int J Occup Saf Ergon 17(4):403-410. https://doi.org/10.1080/ 10803548.2011 .11076903

Zyda M (2005) From visual simulation to virtual reality to games. Computer 38:25-33. https://doi.org/10.1109/mc.2005.297

Publisher's Note Springer Nature remains neutral with regard to jurisdictional claims in published maps and institutional affiliations. 